\title{
Phase transitions for spatially extended pinning
}

\author{
Francesco Caravenna ${ }^{1}$ (D) Frank den Hollander $^{2}$ (D) \\ Dedicated to the memory of Harry Kesten, who for over half a century generously \\ showed the way in probability theory
}

Received: 10 June 2020 / Revised: 11 April 2021 / Accepted: 26 May 2021 / Published online: 9 June 2021

(c) The Author(s) 2021

\begin{abstract}
We consider a directed polymer of length $N$ interacting with a linear interface. The monomers carry i.i.d. random charges $\left(\omega_{i}\right)_{i=1}^{N}$ taking values in $\mathbb{R}$ with mean zero and variance one. Each monomer $i$ contributes an energy $\left(\beta \omega_{i}-h\right) \varphi\left(S_{i}\right)$ to the interaction Hamiltonian, where $S_{i} \in \mathbb{Z}$ is the height of monomer $i$ with respect to the interface, $\varphi: \mathbb{Z} \rightarrow[0, \infty)$ is the interaction potential, $\beta \in[0, \infty)$ is the inverse temperature, and $h \in \mathbb{R}$ is the charge bias parameter. The configurations of the polymer are weighted according to the Gibbs measure associated with the interaction Hamiltonian, where the reference measure is given by a Markov chain on $\mathbb{Z}$. We study both the quenched and the annealed free energy per monomer in the limit as $N \rightarrow \infty$. We show that each exhibits a phase transition along a critical curve in the $(\beta, h)$-plane, separating a localized phase (where the polymer stays close to the interface) from a delocalized phase (where the polymer wanders away from the interface). We derive variational formulas for the critical curves and we obtain upper and lower bounds on the quenched critical curve in terms of the annealed critical curve. In addition, for the special case where the reference measure is given by a Bessel random walk, we derive the scaling limit of the annealed free energy as $\beta, h \downarrow 0$ in three different regimes for the tail exponent of $\varphi$.
\end{abstract}

The research in this paper was supported by ERC Advanced Grant 267356 VARIS and NWO Gravitation Grant 024.002.003 NETWORKS.

$\bowtie \quad$ Frank den Hollander

denholla@math.leidenuniv.nl

Francesco Caravenna

francesco.caravenna@unimib.it

1 Dipartimento di Matematica e Applicazioni, Università degli Studi di Milano-Bicocca, Via Cozzi 55, 20125 Milan, Italy

2 Mathematisch instituut, Universiteit Leiden, Postbus 9512, 2300, RA Leiden, The Netherlands 
Keywords Charged polymers · Spatially extended pinning · Free energy · Critical curve $\cdot$ Phase transition - Large deviation principle · Variational representation

Mathematics Subject Classification $60 \mathrm{~K} 37 \cdot 82 \mathrm{~B} 44 \cdot 82 \mathrm{~B} 41$

\section{Introduction}

\subsection{Motivation}

Homogeneous pinning models, where a directed polymer receives a reward for every monomer that hits an interface, have been the object of intense study. Both discrete and continuous models have been analysed in detail, and a full understanding is available of the free energy, the phase diagram and the typical polymer configurations as a function of the underlying model parameters. Disordered pinning models, where the reward depends on random weights attached to the interface or where the shape of the interface is random itself, are much harder to analyse. Still, a lot of progress has been made in past years, in particular, the effect of the disorder on the scaling properties of the polymer has been elucidated to considerable depth. For an overview the reader is referred to the monographs by Giacomin [21,22] and den Hollander [24], the review paper by Caravenna et al. [13], and references therein.

Spatially extended pinning, where the interaction of the monomers depends on their distance to the interface, remains largely unexplored. For a continuum model with an interaction potential that decays polynomially with the distance, pinning-like results have been obtained in Lacoin [25]. A continuum model for which the interaction potential is non-zero only in a finite window around the interface was analysed in Cranston et al. [18]. The goal of the present paper is to investigate what happens for more general interaction potentials, both for discrete and for continuous models with disorder.

The remainder of this section is organised as follows. In Sect. 1.2 we define our model, which consists of a directed polymer carrying random charges that interact with a linear interface at a strength that depends on their distance. In Sects. 1.3 and 1.4 we look at the quenched, respectively, the annealed free energy, and discuss the qualitative properties of the phase diagram. In Sect. 1.5 we recall certain scaling properties of the Bessel random walk and its relation to the Bessel process, both of which play an important role in our analysis. In Sect. 1.6 we state three theorems when the underlying reference measure (describing the polymer without interaction) is a Markov chain. In Sect. 1.7 we state three theorems for the limit of weak interaction when the reference measure is the Bessel random walk, and show that this limit is related to the continuum version of our model when the reference measure is the Bessel process. In Sect. 1.8 we place the theorems in their proper perspective. In Sect. 1.9 we list some open problems and explain how the proofs of the theorems are organised. 


\subsection{The model}

Let $\mathbb{N}_{0}=\mathbb{N} \cup\{0\}$. Our model has three ingredients:

(1) An irreducible nearest-neighbour recurrent Markov chain $S:=\left(S_{n}\right)_{n \in \mathbb{N}_{0}}$ on $\mathbb{Z}$ starting at $S_{0}=0$, with law $\mathrm{P}=\mathrm{P}_{0}$.

(2) An i.i.d. sequences of random charges $\omega:=\left(\omega_{n}\right)_{n \in \mathbb{N}}$ on $\mathbb{R}$, with law $\mathbb{P}$.

(3) A non-negative function $\varphi: \mathbb{Z} \rightarrow[0, \infty)$, playing the role of an interaction potential, such that

$$
0<\|\varphi\|_{\infty}<\infty, \quad \lim _{x \rightarrow \infty} \varphi(x)=0, \quad \lim _{x \rightarrow-\infty} \varphi(x) \text { exists. }
$$

Our model is defined through the quenched partition function

$$
Z_{N, \beta}^{\omega}:=\mathrm{E}\left[\mathrm{e}^{\beta \sum_{n=1}^{N} \omega_{n} \varphi\left(S_{n}\right)}\right], \quad N \in \mathbb{N}_{0},
$$

which describes a directed polymer chain $n \mapsto\left(n, S_{n}\right)$ of length $N$ carrying charges $n \mapsto \omega_{n}$ that interact with a linear interface according to the interaction potential $x \mapsto \varphi(x)$ at inverse temperature $\beta \in[0, \infty)$. Without loss of generality we may replace $\beta \omega_{n}$ by $\beta \omega_{n}-h$, with $h \in \mathbb{R}$ the charge bias parameter, and assume that $\omega$ is standardized, i.e.,

$$
\mathbb{E}\left[\omega_{n}\right]=0, \quad \mathbb{V a r}\left[\omega_{n}\right]=1,
$$

after which (1.2) becomes

$$
Z_{N, \beta, h}^{\omega}:=\mathrm{E}\left[\mathrm{e}^{\sum_{n=1}^{N}\left(\beta \omega_{n}-h\right) \varphi\left(S_{n}\right)}\right], \quad N \in \mathbb{N}_{0} .
$$

Throughout the sequel we assume that

$$
\mathrm{M}(t):=\mathbb{E}\left[\mathrm{e}^{t \omega_{1}}\right]<\infty \quad \forall t \in \mathbb{R}
$$

Moreover, defining $\tau_{1}:=\inf \left\{n \in \mathbb{N}: S_{n}=0\right\}$ to be the first return time of $S$ to 0 , we assume that there exists an $\alpha \in[0, \infty)$ such that

$$
\sum_{n \in \mathbb{N}} \mathrm{P}\left(\tau_{1}=n\right)=1, \quad \mathrm{P}\left(\tau_{1}=n\right)=n^{-(1+\alpha)+o(1)}, \quad n \rightarrow \infty
$$

Note that $\mathrm{E}\left(\tau_{1}\right)=\infty$ for all $\alpha \in(0,1)$. If $S$ has period 2 , then the last asymptotics is assumed to run along $2 \mathbb{N}$.

Remark 1.1 (Bessel random walk). An example of a Markov chain $S$ satisfying (1.6) that will receive special attention in this paper is the one with transition probabilities

$$
\mathrm{P}\left(S_{n+1}=x \pm 1 \mid S_{n}=x\right)=: \frac{1}{2}[1 \pm d(x)], \quad x \in \mathbb{Z},
$$


where

$$
d(x)=-d(-x), \quad x \in \mathbb{Z}, \quad d(x)=-\left(\alpha-\frac{1}{2}\right) x^{-1}+O\left(|x|^{-(1+\varepsilon)}\right), \quad|x| \rightarrow \infty,
$$

for some $\alpha \in(0,1)$ and $\varepsilon>0$. This choice, which is referred to as the Bessel random walk, has a drift away from the origin $\left(\alpha<\frac{1}{2}\right)$ or towards the origin $\left(\alpha>\frac{1}{2}\right)$ that decays inversely proportional to the distance. The case $d(x) \equiv 0\left(\alpha=\frac{1}{2}\right)$ corresponds to simple random walk. The Bessel random walk was studied by Lamperti [26] and, more recently, by Alexander [2] (who actually considered the one-sided version $\left(\left|S_{n}\right|\right)_{n \in \mathbb{N}_{0}}$ ). It is known that (1.6) holds in a sharp form [2, Theorem 2.1], namely,

$$
\mathrm{P}\left(\tau_{1}=n\right) \sim c n^{-(1+\alpha)}, \quad n \rightarrow \infty,
$$

along $2 \mathbb{N}$ for some $c \in(0, \infty)$. More refined asymptotics are available as well (see Sect. 1.5 below).

Remark 1.2 The model defined in (1.4) provides a natural interpolation between the pinning model and the copolymer model, which correspond to the choices

$$
\varphi^{\mathrm{pin}}(x)=1_{\{x=0\}}, \quad \varphi^{\mathrm{cop}}(x)=1_{\{x \leq 0\}} .
$$

See Giacomin [21,22] and den Hollander [24] for details. Actually, in the copolymer model the interaction is via the bonds rather than the sites of the path, i.e., $\varphi^{\mathrm{cop}}((x, y))=1_{\{x+y \leq 0\}}$, but we will ignore such refinements. Moreover, the standard parametrisation of the disorder in the copolymer model is $-2 \beta\left(\omega_{n}+h\right)$ rather than $\beta \omega_{n}-h$. Again, this is the same after a change of parameters. Our choice has the advantage that the free energy is jointly convex in $(\beta, h)$ and that the critical curve is non-negative (see Fig. 1).

\subsection{The quenched free energy}

The quenched free energy is defined by

$$
\mathrm{F}^{\text {que }}(\beta, h):=\lim _{N \rightarrow \infty} \frac{1}{N} \log Z_{N, \beta, h}^{\omega} \quad \mathbb{P} \text {-a.s. and in } L^{1}(\mathbb{P}) \text {. }
$$

For the constrained partition function

$$
Z_{N, \beta, h}^{\omega, \mathrm{c}}:=\mathrm{E}\left[\mathrm{e}^{\sum_{n=1}^{N}\left(\beta \omega_{n}-h\right) \varphi\left(S_{n}\right)} 1_{\left\{S_{N}=0\right\}}\right], \quad N \in \mathbb{N}_{0},
$$

the existence of the limit

$$
\lim _{N \rightarrow \infty} \frac{1}{N} \log Z_{N, \beta, h}^{\omega, \mathrm{c}} \quad \mathbb{P} \text {-a.s. and in } L^{1}(\mathbb{P})
$$


follows by standard super-additivity arguments. Since $\varphi$ is bounded and $\mathbb{P}$ has finite exponential moments (recall (1.5)), the limit is finite. We will show in "Appendix A.2" that

$$
\lim _{N \rightarrow \infty} \frac{1}{N} \log \frac{Z_{N, \beta, h}^{\omega, \mathrm{c}}}{Z_{N, \beta, h}^{\omega}}=0 \quad \mathbb{P} \text {-a.s. and in } L^{1}(\mathbb{P}),
$$

so that (1.11) follows.

By (1.1), for every $\varepsilon>0$ there is an $M \in \mathbb{N}_{0}$ such that $0 \leq \varphi(x) \leq \varepsilon$ for $x \geq M$. Therefore

$$
\begin{gathered}
Z_{N, \beta, h}^{\omega, \mathrm{c}} \geq \mathrm{e}^{-\|\varphi\|_{\infty} \sum_{n=1}^{M-1}\left(\beta\left|\omega_{n}\right|+|h|\right)-\varepsilon \sum_{n=M}^{N}\left(\beta\left|\omega_{n}\right|+|h|\right)} \mathrm{P}\left(S_{n} \geq M \forall M<n \leq N\right) \\
\forall M \in \mathbb{N}_{0} .
\end{gathered}
$$

We will show in "Appendix A.1" that, by (1.6),

$$
\lim _{N \rightarrow \infty} \frac{1}{N} \log \mathrm{P}\left(S_{n} \geq M \forall M<n \leq N\right)=0 \quad \forall M \in \mathbb{N}_{0}
$$

and so it follows that $\mathrm{F}^{\text {que }}(\beta, h) \geq-\varepsilon\left(\beta \mathbb{E}\left[\left|\omega_{1}\right|\right]+|h|\right)$. Since $\varepsilon>0$ is arbitrary, we obtain the important inequality

$$
\mathrm{F}^{\text {que }}(\beta, h) \geq 0 \quad \forall \beta \in[0, \infty), h \in \mathbb{R} \text {. }
$$

It is therefore natural to define the two phases

$$
\begin{aligned}
& \mathcal{L}^{\text {que }}:=\left\{(\beta, h): \mathrm{F}^{\text {que }}(\beta, h)>0\right\}, \\
& \mathcal{D}^{\text {que }}:=\left\{(\beta, h): \mathrm{F}^{\text {que }}(\beta, h)=0\right\},
\end{aligned}
$$

which we refer to as the quenched localized phase, respectively, the quenched delocalized phase.

By $(1.11),(\beta, h) \mapsto \mathrm{F}^{\text {que }}(\beta, h)$ is the pointwise limit of jointly convex functions. Moreover, $h \mapsto Z_{N, \beta, h}^{\omega}$ is non-increasing, so that $h \mapsto \mathrm{F}^{\text {que }}(\beta, h)$ is non-increasing as well. Furthermore, $\beta \mapsto \mathbb{E}\left[\log Z_{N, \beta, h}^{\omega}\right]$ is convex and (by direct computation) has zero derivative at $\beta=0$, so that $\beta \mapsto \mathrm{F}^{\text {que }}(\beta, h)$ is non-decreasing on $[0, \infty)$.

From the monotonicity of $h \mapsto \mathrm{F}^{\text {que }}(\beta, h)$ it follows that $\mathcal{L}^{\text {que }}$ and $\mathcal{D}^{\text {que }}$ are separated by a quenched critical curve $h_{c}^{\text {que }}:[0, \infty) \rightarrow[0, \infty)$ whose graph is $\partial \mathcal{D}^{\text {que }}$ (see Fig. 1):

$$
\begin{aligned}
& \mathcal{L}^{\text {que }}:=\left\{(\beta, h): h<h_{c}^{\text {que }}(\beta)\right\}, \\
& \mathcal{D}^{\text {que }}:=\left\{(\beta, h): h \geq h_{c}^{\text {que }}(\beta)\right\} .
\end{aligned}
$$

From the convexity of $(\beta, h) \mapsto \mathrm{F}^{\text {que }}(\beta, h)$ it follows that the lower level set $\mathcal{D}^{\text {que }}=$ $\left\{(\beta, h): \mathrm{F}^{\text {que }}(\beta, h) \leq 0\right\}$ is convex. Since $\mathcal{D}^{\text {que }}$ is the upper graph of $h_{c}^{\text {que }}$, it follows that $h_{c}^{\text {que }}$ is convex and hence continuous. In Sect. 1.6 we will see that $h_{c}^{\text {que }}$ is finite 
Fig. 1 Qualitative plot of $\beta \mapsto h_{c}^{\text {que }}(\beta)$

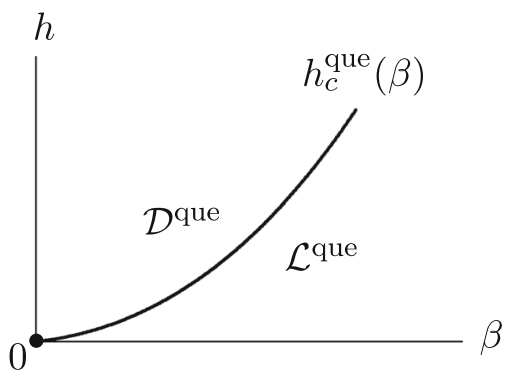

everywhere. Since $S$ is recurrent, it follows from the theory of the homogeneous pinning model (Giacomin [21,22], den Hollander [24]) that $\mathrm{F}^{\text {que }}(0, h)>0$ for $h<0$ and $\mathrm{F}^{\text {que }}(0, h)=0$ for $h \geq 0$. Hence $h_{c}^{\text {que }}(0)=0$. (Note that $\varphi(x) \geq \varphi\left(x_{*}\right) 1_{\left\{x_{*}\right\}}(x)$ for any $x_{*} \in \mathbb{Z}$ with $\varphi\left(x_{*}\right)>0$. Therefore we can dominate the quenched free energy for $\beta=0$ by the free energy of the homogeneous pinning model with a strictly positive pinning reward.)

Finally, from the monotonicity of $\beta \mapsto \mathrm{F}^{\text {que }}(\beta, h)$ on $[0, \infty)$ it follows that $\mathrm{F}^{\text {que }}(\beta, h) \geq \mathrm{F}^{\text {que }}(0, h)>0$ for $h<0$, so that $h_{c}^{\text {que }}(\beta) \geq h_{c}^{\text {que }}(0)=0$ for $\beta \geq 0$. Since $h_{c}^{\text {que }}$ is convex, this implies that $h_{c}^{\text {que }}$ is non-decreasing, and is strictly increasing as soon as it leaves zero. In Sect. 1.6 we will see that $h_{c}^{\text {que }}(\beta)>0$ for all $\beta>0$ (see Fig. 1).

\subsection{The annealed free energy}

The annealed partition function associated with (1.4) is

$$
Z_{N, \beta, h}^{\mathrm{ann}}:=\mathbb{E}\left[Z_{N, \beta, h}^{\omega}\right]=\mathrm{E}\left[\mathrm{e}^{\sum_{n=1}^{N} \psi_{\beta, h}\left(S_{n}\right)}\right], \quad N \in \mathbb{N}_{0}
$$

where

$$
\psi_{\beta, h}(x):=\log \mathrm{M}(\beta \varphi(x))-h \varphi(x)
$$

This is the partition function of the homogeneous pinning model with potential $\psi_{\beta, h}$. A delicate point is that $\psi_{\beta, h}$ does not have a sign: it may be a mixture of attractive and repulsive interactions. This comes from the fact that both the charge distribution and the interaction potential are general.

The annealed free energy is defined by

$$
\mathrm{F}^{\mathrm{ann}}(\beta, h):=\lim _{N \rightarrow \infty} \frac{1}{N} \log Z_{N, \beta, h}^{\mathrm{ann}} .
$$

For the constrained partition function

$$
Z_{N, \beta, h}^{\mathrm{ann}, \mathrm{c}}:=\mathrm{E}\left[\mathrm{e}^{\sum_{n=1}^{N} \psi_{\beta, h}\left(S_{n}\right)} 1_{\left\{S_{N}=0\right\}}\right], \quad N \in \mathbb{N}_{0},
$$


Fig. 2 Qualitative plot of $\beta \mapsto h_{c}^{\mathrm{ann}}(\beta)$

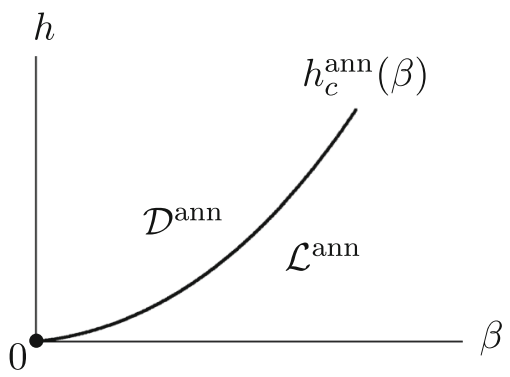

the existence of the limit

$$
\lim _{N \rightarrow \infty} \frac{1}{N} \log Z_{N, \beta, h}^{\mathrm{ann}, \mathrm{c}}
$$

again follows by standard super-additivity arguments. Since $\psi_{\beta, h}$ is bounded, the limit is finite. The analogue of (1.14), which will be proved in Appendix A.3, reads

$$
\lim _{N \rightarrow \infty} \frac{1}{N} \log \frac{Z_{N, \beta, h}^{\mathrm{ann}, \mathrm{c}}}{Z_{N, \beta, h}^{\mathrm{ann}}}=0,
$$

so that (1.22) follows.

The annealed localized phase, annealed delocalized phase and annealed critical curve are defined as

$$
\begin{aligned}
& \mathcal{L}^{\mathrm{ann}}:=\left\{(\beta, h): \mathrm{F}^{\mathrm{ann}}(\beta, h)>0\right\}=\left\{(\beta, h): h<h_{c}^{\mathrm{ann}}(\beta)\right\}, \\
& \mathcal{D}^{\mathrm{ann}}:=\left\{(\beta, h): \mathrm{F}^{\mathrm{ann}}(\beta, h)=0\right\}=\left\{(\beta, h): h \geq h_{c}^{\mathrm{ann}}(\beta)\right\} .
\end{aligned}
$$

As is clear from (1.21) and the fact that $\varphi$ is non-negative, $\mathrm{F}^{\mathrm{ann}}(\beta, h)$ is non-increasing and convex as a function of $h$, and non-decreasing as a function of $\beta$ but not necessarily convex. Later we will see that nonetheless $\beta \mapsto h_{c}^{\text {ann }}(\beta)$ has a shape that is qualitatively similar to that of $\beta \mapsto h_{c}^{\text {que }}(\beta)$ (see Fig. 2).

An important property of the annealed free energy is that it provides an upper bound for the quenched free energy: by Jensen's inequality we have $\mathrm{F}^{\text {que }}(\beta, h) \leq \mathrm{F}^{\mathrm{ann}}(\beta, h)$ for all $\beta \in[0, \infty)$ and $h \in \mathbb{R}$. Recalling (1.17), we therefore see that

$$
0 \leq h_{c}^{\text {que }}(\beta) \leq h_{c}^{\text {ann }}(\beta) \quad \forall \beta \geq 0 .
$$

Unlike for the pinning model and the copolymer model, for general potentials $\varphi$ the annealed free energy and the annealed critical curve are not known explicitly.

\subsection{Scaling properties of the Bessel random walk}

Part of our results below involve the annealed free energy and the annealed critical curve associated with a Brownian version of the model, where the reference measure is 
based on the Bessel process $X:=\left(X_{t}\right)_{t \geq 0}$ of dimension $2(1-\alpha)($ see $[27$, Chapter XI]) defined by

$$
\mathrm{d} X_{t}=\mathrm{d} B_{t}-\frac{\alpha-\frac{1}{2}}{X_{t}} \mathrm{~d} t \quad \text { on }[0, \infty) \text { with reflection at } 0
$$

where $\alpha \in(0,1)$ and $\left(B_{t}\right)_{t \geq 0}$ is standard Brownian motion on $\mathbb{R} .{ }^{1}$ Informally, $X$ makes infrequent visits to 0 when $\alpha<\frac{1}{2}$ and frequent visits to 0 when $\alpha>\frac{1}{2}$. The choice $\alpha=\frac{1}{2}$ corresponds to reflected Brownian motion, i.e., $X_{t}=\left|B_{t}\right|$ with $\left(B_{t}\right)_{t \geq 0}$ standard Brownian motion. We write $\hat{\mathrm{P}}_{x}$ to denote the law of $X$ given $X_{0}=x$. When $x=0$, we simply write $\hat{\mathrm{P}}=\hat{\mathrm{P}}_{0}$.

For the semigroup $g_{t}(x, y):=\hat{\mathrm{P}}_{x}\left(X_{t} \in \mathrm{d} y\right) / \mathrm{d} y$ of $X$ there is an explicit formula, namely,

$$
g_{t}(x, y)=\frac{x^{\alpha} y^{1-\alpha}}{t} \mathrm{e}^{-\frac{x^{2}+y^{2}}{2 t}} J_{-\alpha}\left(\frac{x y}{t}\right), \quad x, y \in(0, \infty), \quad t>0,
$$

where $J_{-\alpha}(z)=\sum_{m \in \mathbb{N}_{0}} \frac{(-1)^{m}}{m ! \Gamma(m+1-\alpha)}\left(\frac{z}{2}\right)^{2 m-\alpha}$ is the Bessel function of index $-\alpha$. Since

$$
J_{-\alpha}(z) \sim \frac{2^{\alpha}}{\Gamma(1-\alpha)} z^{-\alpha}, \quad z \downarrow 0,
$$

we also have the explicit formula

$g_{t}(y):=g_{t}(0, y):=\frac{\hat{\mathrm{P}}\left(X_{t} \in \mathrm{d} y\right)}{\mathrm{d} y}=\frac{2^{\alpha}}{\Gamma(1-\alpha)} \frac{y^{1-2 \alpha}}{t^{1-\alpha}} \mathrm{e}^{-\frac{y^{2}}{2 t}}, \quad y \in[0, \infty), \quad t>0$.

It follows that

$$
\hat{\mathrm{P}}\left(X_{t}<\varepsilon\right) \leq \frac{\varepsilon^{2(1-\alpha)}}{c_{\alpha} t^{1-\alpha}}, \quad t, \varepsilon>0, \quad c_{\alpha}:=\frac{\Gamma(2-\alpha)}{2^{\alpha-1}},
$$

and this inequality becomes sharp as $\varepsilon \downarrow 0$, namely,

$$
\lim _{\varepsilon \downarrow 0} \frac{c_{\alpha}}{\varepsilon^{2(1-\alpha)}} \hat{\mathrm{P}}_{x}\left(X_{t}<\varepsilon\right)=\lim _{\varepsilon \downarrow 0} \frac{c_{\alpha}}{\varepsilon^{2(1-\alpha)}} \int_{0}^{\varepsilon} g_{t}(x, y) \mathrm{d} y=\hat{g}_{t}(x, 0), \quad x \geq 0,
$$

where

$$
\hat{g}_{t}(x, 0):=\frac{1}{t^{1-\alpha}} \mathrm{e}^{-\frac{x^{2}}{2 t}}, \quad x \in[0, \infty), \quad t>0
$$

\footnotetext{
${ }^{1}$ Formally, the squared Bessel process $\left(Y_{t}\right)_{t \geq 0}$ is defined by $\mathrm{d} Y_{t}=2 \sqrt{Y_{t}} \mathrm{~d} B_{t}+2(1-\alpha) \mathrm{d} t$ on $[0, \infty)$ with reflection at 0 , and $\left(X_{t}\right)_{t \geq 0}$ is defined by setting $X_{t}=\sqrt{Y_{t}}$. 
The local time of $X$ at 0 up to time $T \geq 0$ is defined as the following limit in probability:

$$
\hat{L}_{T}(0):=\lim _{\varepsilon \downarrow 0} \frac{c_{\alpha}}{\varepsilon^{2(1-\alpha)}} \int_{0}^{T} \mathrm{~d} t 1_{(0, \varepsilon)}\left(X_{t}\right) .
$$

We will informally write

$$
\hat{L}_{T}(0)=: \int_{0}^{T} \mathrm{~d} t \hat{\delta}_{0}\left(X_{t}\right)
$$

Note that $\hat{\mathrm{E}}\left[\hat{L}_{T}(0)\right]=\int_{0}^{T} \frac{\mathrm{d} t}{t^{1-\alpha}}=\int_{0}^{T} \hat{g}_{t}(0,0) \mathrm{d} t=\frac{1}{\alpha} T^{\alpha}$.

The relation of the Bessel process with the Bessel random walk defined in Remark 1.1 is that the latter satisfies the invariance principle (see Lamperti [26])

$$
\left(\left|S_{\lfloor N t\rfloor}\right| / \sqrt{N}\right)_{t \geq 0} \Longrightarrow\left(X_{t}\right)_{t \geq 0}, \quad N \rightarrow \infty
$$

Write $\mathrm{P}_{k^{\prime}}(\cdot):=\mathrm{P}\left(\cdot \mid S_{0}=k^{\prime}\right)$ to denote the law of the Bessel random walk started at $k^{\prime} \in \mathbb{Z}$, so that $\mathrm{P}=\mathrm{P}_{0}$. Local limit theorems for the transition probabilities $\mathrm{P}_{k^{\prime}}\left(\left|S_{n}\right|=\right.$ $k$ ) of the Bessel random walk have been established in [2, Theorem 2.4]. The following formulas hold in the limit as $n \rightarrow \infty$, uniformly in a specified range of $k, k^{\prime} \in \mathbb{N}_{0}$. We assume that $k-k^{\prime}$ has the same parity as $n$, i.e., $k-k^{\prime}-n$ is even, because otherwise $\mathrm{P}_{k^{\prime}}\left(\left|S_{n}\right|=k\right)=0$.

- Low ending heights: For any $\varepsilon>0$ and $\bar{k}_{n}=o(\sqrt{n})$, uniformly in $0 \leq k^{\prime} \leq \sqrt{n} / \varepsilon$ and $0 \leq k \leq \bar{k}_{n}$,

$$
\mathrm{P}_{k^{\prime}}\left(\left|S_{n}\right|=k\right) \sim \frac{2 c(k)}{n^{1-\alpha}} \hat{g}_{1}\left(\frac{k^{\prime}}{\sqrt{n}}, 0\right) 1_{\left\{k-k^{\prime}-n \text { is even }\right\}}, \quad n \rightarrow \infty
$$

where $\hat{g}_{1}$ is defined in (1.34) and $c: \mathbb{N}_{0} \rightarrow(0, \infty)$ is an explicit function (which depends on the function $d: \mathbb{Z} \rightarrow \mathbb{R}$ in (1.7)) such that

$$
c(k) \sim \frac{2^{\alpha}}{\Gamma(1-\alpha)} k^{1-2 \alpha}, \quad k \rightarrow \infty
$$

In case of a low starting height $k^{\prime}=o(\sqrt{n}),(1.38)$ simplifies because $\hat{g}_{1}\left(\frac{k^{\prime}}{\sqrt{n}}, 0\right) \sim$ $\hat{g}_{1}(0,0)=1$.

- Intermediate ending height: For any $\varepsilon>0$, uniformly in $0 \leq k^{\prime} \leq \sqrt{n} / \varepsilon$ and $\varepsilon \sqrt{n} \leq k \leq \sqrt{n} / \varepsilon$,

$$
\mathrm{P}_{k^{\prime}}\left(\left|S_{n}\right|=k\right) \sim \frac{2}{\sqrt{n}} g_{1}\left(\frac{k^{\prime}}{\sqrt{n},} \frac{k}{\sqrt{n}}\right) 1_{\left\{k-k^{\prime}-n \text { is even }\right\}}, \quad n \rightarrow \infty
$$


where $g_{1}$ is the density in (1.29) at time 1 . In case of a low starting height $k^{\prime}=$ $o(\sqrt{n}),(1.38)$ simplifies because $g_{1}\left(\frac{k^{\prime}}{\sqrt{n}}, \frac{k}{\sqrt{n}}\right) \sim g_{1}\left(0, \frac{k}{\sqrt{n}}\right)=g_{1}\left(\frac{k}{\sqrt{n}}\right)$ reduces to (1.31).

- High ending heights: There exists a $C=C(\alpha)<\infty$ such that, for all $k \geq \sqrt{n}$,

$$
\mathrm{P}\left(\left|S_{n}\right|=k\right) \leq \frac{C}{\sqrt{n}} \mathrm{e}^{-\frac{k^{2}}{8 n}} 1_{\{k-n \text { is even }\}} .
$$

It follows from (1.38)-(1.41) that, for some $C<\infty$,

$$
\forall n \in \mathbb{N} \quad \forall k \in \mathbb{Z}: \quad \mathrm{P}\left(\left|S_{n}\right|=k\right) \leq C \frac{(1+|k|)^{1-2 \alpha}}{n^{1-\alpha}} e^{-\frac{k^{2}}{8 n}} 1_{\{k-n \text { is even }\}} .
$$

This uniform bound will be needed to control scaling computations.

Remark 1.3 Equations (1.38) and (1.40) for $k^{\prime}=0$ are proved in [2, Theorem 2.4], while the case $k^{\prime} \neq 0$ follows via the relation $\mathrm{P}_{k^{\prime}}\left(\left|S_{n}\right|=k\right)=\sum_{m=1}^{n} \mathrm{P}_{k^{\prime}}\left(\tau_{1}=\right.$ m) $\mathrm{P}_{0}\left(\left|S_{n-m}\right|=k\right)+O\left(\mathrm{P}_{k^{\prime}}\left(\tau_{1}>n\right)\right.$ ) (see also the estimates on $\mathrm{P}_{k^{\prime}}\left(\tau_{1}=m\right.$ ) provided in [2, Theorem 2.2]). We further point out the duality relation $\mathrm{P}_{k^{\prime}}\left(\left|S_{n}\right|=k\right)=$ $\frac{1+d\left(k^{\prime}\right)}{1+d(k)} \frac{\lambda_{k^{\prime}}}{\lambda_{k}} \mathrm{P}_{k}\left(\left|S_{n}\right|=k^{\prime}\right)$, where $\lambda_{k}:=\prod_{x=1}^{k} \frac{1-d(x)}{1+d(x)}$.

\subsection{General properties}

Our first set of theorems concerns the quenched and the annealed critical curve.

Theorem 1.4 $\beta \mapsto h_{c}^{\text {que }}(\beta)$ and $\beta \mapsto h_{c}^{\text {ann }}(\beta)$ can be characterized in terms of variational formulas (see Theorems 2.4-2.5 below).

Theorem 1.5 For every $\beta \geq 0$,

$$
(1+\alpha) h_{c}^{\mathrm{ann}}\left(\frac{\beta}{1+\alpha}\right) \leq h_{c}^{\mathrm{que}}(\beta) \leq h_{c}^{\mathrm{ann}}(\beta) .
$$

As already noted in (1.27), the second inequality in (1.43) is an immediate consequence of Jensen's inequality applied to (1.12) and (1.23). The first inequality in (1.43), which is known as the Monthus-Bodineau-Giacomin bound, was previously shown to hold for the copolymer model [9], [11]. We show that it holds for the general class of potentials satisfying (1.1).

In Sect. 3.3 we will show that $F^{\text {ann }}(\beta, 0)>0$ for all $\beta>0$. This implies that $h_{c}^{\text {ann }}(\beta)>0$ for all $\beta>0$, which via (1.43) settles the claim made at the end of Sect. 1.3 that $h_{c}^{\text {que }}(\beta)>0$ for all $\beta>0$.

\subsection{Scaling for weak interaction}

Our second set of theorems looks at the scaling of the annealed free energy in the limit of weak interaction, for the special case where $S$ is the Bessel random walk 
with parameter $\alpha \in(0,1)$ defined in Remark 1.1 and the interaction potential $\varphi$ is symmetric: $\varphi(-x)=\varphi(x)$ for all $x \in \mathbb{Z}$. We consider three different regimes for the tail behaviour of $\varphi$, namely,

$$
\lim _{|x| \rightarrow \infty}|x|^{\vartheta} \varphi(x)=c \in(0, \infty)
$$

with

$$
\begin{aligned}
& \vartheta \in(0,1-\alpha), \\
& \vartheta \in(1-\alpha, 2(1-\alpha)), \\
& \vartheta \in(2(1-\alpha), \infty) .
\end{aligned}
$$

Theorem 1.6 Suppose that $\alpha \in(0,1)$ and $\vartheta \in(0,1-\alpha)$. For every $\hat{\beta} \in(0, \infty)$ and $\hat{h} \in(0, \infty)$,

$$
\lim _{\delta \downarrow 0} \delta^{-1} F^{\text {ann }}\left(\hat{\beta}, \delta^{(1-\vartheta) / 2}, \hat{h} \delta^{(2-\vartheta) / 2}\right)=\hat{F}^{\text {ann }}(\hat{\beta}, \hat{h})
$$

where

$$
\hat{F}^{\mathrm{ann}}(\hat{\beta}, \hat{h}):=\lim _{T \rightarrow \infty} \frac{1}{T} \log \hat{\mathrm{E}}\left[\exp \left(\frac{1}{2} \hat{\beta}^{2} c^{2} \int_{0}^{T} d t X_{t}^{-2 \vartheta}-\hat{h} c \int_{0}^{T} d t X_{t}^{-\vartheta}\right)\right]
$$

with $c$ the constant in (1.44).

Theorem 1.7 Suppose that $\alpha \in(0,1)$ and $\vartheta \in(1-\alpha, 2(1-\alpha))$. For every $\hat{\beta} \in(0, \infty)$ and $\hat{h} \in(0, \infty)$,

$$
\lim _{\delta \downarrow 0} \delta^{-1} F^{\mathrm{ann}}\left(\hat{\beta}, \delta^{\alpha / 2}, \hat{h} \delta^{(2-\vartheta) / 2}\right)=\hat{F}^{\mathrm{ann}}(\hat{\beta}, \hat{h}),
$$

where

$$
\hat{F}^{\mathrm{ann}}(\hat{\beta}, \hat{h}):=\lim _{T \rightarrow \infty} \frac{1}{T} \log \hat{\mathrm{E}}\left[\exp \left(\frac{1}{2} \hat{\beta}^{2} c^{*}\left[\varphi^{2}\right] \hat{L}_{T}(0)-\hat{h} c \int_{0}^{T} d t X_{t}^{-\vartheta}\right)\right]
$$

with $c$ the constant in (1.44) and $c^{*}\left[\varphi^{2}\right]=\sum_{x \in \mathbb{Z}} \varphi^{2}(x) c(x)$, where $x \mapsto c(x):=$ $c(|x|)$ is the function in (1.38)-(1.39).

Theorem 1.8 Suppose that $\alpha \in(0,1)$ and $\vartheta \in(2(1-\alpha), \infty)$. For every $\hat{\beta} \in(0, \infty)$ and $\hat{h} \in(0, \infty)$,

$$
\lim _{\delta \downarrow 0} \delta^{-1} F^{\text {ann }}\left(\hat{\beta}, \delta^{\alpha / 2}, \hat{h} \delta^{\alpha}\right)=\hat{F}^{\text {ann }}(\hat{\beta}, \hat{h}),
$$


Fig. 3 Plot of $\hat{\beta} \mapsto \hat{h}_{c}^{\text {ann }}(\hat{\beta})$ in (1.53)

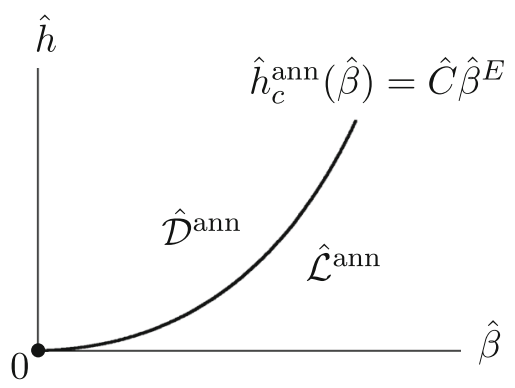

where

$$
\hat{F}^{\mathrm{ann}}(\hat{\beta}, \hat{h}):=\lim _{T \rightarrow \infty} \frac{1}{T} \log \hat{\mathrm{E}}\left[\exp \left(\left\{\frac{1}{2} \hat{\beta}^{2} c^{*}\left[\varphi^{2}\right]-\hat{h} c^{*}[\varphi]\right\} \hat{L}_{T}(0)\right)\right]
$$

with $c^{*}[\varphi]=\sum_{x \in \mathbb{Z}} \varphi(x) c(x)$, where $x \mapsto c(x):=c(|x|)$ is the function in (1.38)(1.39).

Note that, because of (1.39) and (1.44), $c^{*}\left[\varphi^{2}\right]<\infty$ when $\vartheta>1-\alpha$ and $c^{*}[\varphi]<$ $\infty$ when $\vartheta>2(1-\alpha)$. In Appendix B.1 we will show that the annealed partition functions associated with the Bessel process appearing in Theorems 1.6-1.8 are finite, and so are the corresponding annealed free energies.

The annealed free energy $\hat{F}^{\text {ann }}(\hat{\beta}, \hat{h})$ appearing in Theorems $1.6-1.8$ has its own phase diagram, with phases

$$
\begin{aligned}
& \mathcal{L}^{\text {ân }}:=\left\{(\hat{\beta}, \hat{h}): \hat{F}^{\text {ann }}(\hat{\beta}, \hat{h})>0\right\}, \\
& \mathcal{D}^{\text {ânn }}:=\left\{(\hat{\beta}, \hat{h}): \hat{F}^{\mathrm{ann}}(\hat{\beta}, \hat{h})=0\right\},
\end{aligned}
$$

and with a critical curve that is a perfect power law (see Fig. 3), namely,

$$
\hat{h}_{c}^{\mathrm{ann}}(\hat{\beta})=\hat{C} \hat{\beta}^{E}, \quad \hat{\beta} \in(0, \infty)
$$

where

$$
E=E(\alpha, \vartheta)= \begin{cases}(2-\vartheta) /(1-\vartheta), & \vartheta \in(0,1-\alpha), \\ (2-\vartheta) / \alpha, & \vartheta \in(1-\alpha, 2(1-\alpha)), \\ 2, & \vartheta \in(2(1-\alpha), \infty),\end{cases}
$$

plays the role of a critical exponent (see Fig. 4). The scaling of the annealed critical curve in Theorems $1.6-1.8$ can be summarised as saying that $h_{c}^{\mathrm{ann}}(\beta) \sim \hat{h}_{c}^{\mathrm{ann}}(\beta)$, $\beta \downarrow 0$.

The constant $\hat{C}$ depends on $\alpha, \varphi$ and can be characterized as the unique solution $\hat{C} \in(0, \infty)$ of the equation $\hat{F}^{\mathrm{ann}}(1, \hat{C})=0$. This constant is hard to identify in the first two regimes. In the third regime $\vartheta \in(2(1-\alpha), \infty)$ it is found by inserting (1.53) 
Fig. 4 Plot of the critical exponent $E$ in (1.54) as a function of $\vartheta$ for fixed $\alpha$. The three regimes for $\vartheta$ are indicated. No information is available at the two crossover points

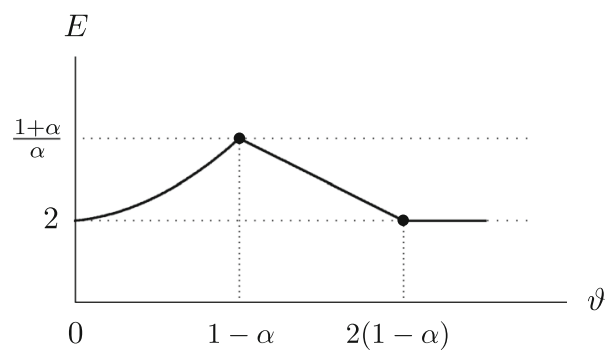

into the equation

$$
\Delta\left(\hat{\beta}, \hat{h}_{c}^{\mathrm{ann}}(\hat{\beta})\right)=0 \quad \text { with } \quad \Delta(\hat{\beta}, \hat{h}):=\frac{1}{2} \hat{\beta}^{2} c^{*}\left[\varphi^{2}\right]-\hat{h} c^{*}[\varphi],
$$

which gives

$$
\hat{C}=\frac{c^{*}\left[\varphi^{2}\right]}{2 c^{*}[\varphi]} .
$$

We show in "Appendix B.2" that, for the the third regime $\vartheta \in(2(1-\alpha), \infty)$, the annealed free energy $\hat{F}^{\text {ann }}(\hat{\beta}, \hat{h})$ can be computed explicitly, namely,

$$
\hat{F}^{\mathrm{ann}}(\hat{\beta}, \hat{h})=(\Gamma(\alpha)[0 \vee \Delta(\hat{\beta}, \hat{h})])^{1 / \alpha} .
$$

Remark 1.9 In view of the scaling limit for the annealed free energies described in Theorems 1.6-1.8, it is natural to expect a scaling limit for the corresponding annealed critical curves as well. Indeed, the continuum critical curve is the perfect power law in (1.53), where $\hat{C}$ and $E$ depend on $\alpha$ and $\varphi$ (and hence on $\vartheta$ ). We conjecture that (1.53) captures the asymptotic behaviour for weak interaction of the discrete critical curve $h_{c}^{\text {ann }}(\beta)$ as well, in the sense that in all three regimes we should have

$$
\lim _{\beta \downarrow 0} \beta^{-E} h_{c}^{\text {ann }}(\beta)=\hat{C} .
$$

This scaling relation cannot be simply deduced from Theorems 1.6-1.8, because pointwise convergence of the free energies does not imply convergence of their zero-level sets, of which the critical curves are the boundaries. However, half of (1.58) follows because if the continuum free energy is strictly positive, then the rescaled discrete free energy eventually becomes strictly positive too in the weak interaction limit, which leads to

$$
\liminf _{\beta \downarrow 0} \beta^{-E} h_{c}^{\mathrm{ann}}(\beta) \geq \hat{C}
$$

In order to prove (1.58) extra work is needed: the scaling of the free energies in (1.46), (1.48) and (1.50) must be strengthened to a perturbative scaling, as shown in [10,12] for the copolymer model and in [16] for the pinning model. 


\subsection{Discussion}

We comment on the results in Sects. 1.6-1.7.

1. The results in Theorems 1.4-1.5 are known for the special case where the interaction potential is that of the pinning model or the copolymer model defined in (1.10). However, the techniques used for these two cases do not carry over to the general class of potentials considered in (1.1). Intuitively, the reason why extension is possible is that the conditions stated in (1.1) say that, outside a large interval around the origin in $\mathbb{Z}$, the interaction potential is controlled by a multiple of that of the copolymer model.

2. As we will see in Sect. 2, the variational formula for $h_{c}^{\text {que }}(\beta)$ mentioned in Theorem 1.4 involves a supremum over the space of all shift-invariant probability distributions on the set of infinite sequences of words of arbitrary length drawn from an infinite sequence of letters taking values in $\mathbb{R} \times \mathbb{Z}$. The supremum involves a quenched rate function that captures the complexity of the interplay between the disorder of the charges and the excursions of the polymer away from the interface. This variational formula is hard to manipulate, but it is the starting point for the proof of Theorems 1.5. The variational formula for $h_{c}^{\mathrm{ann}}(\beta)$ mentioned in Theorem 1.4 is simpler, but still not easy to manipulate (see (1.62) below).

3. Note that for $h=0$ and $\beta>0$ the annealed partition function $Z_{N, \beta, h}^{\mathrm{ann}}$ is bounded from below by the partition function of a homogenous pinning model with a strictly positive reward, which is localized. The lower bound in Theorem 1.5 therefore shows that $h_{c}^{\text {que }}(\beta)>0$ for every $\beta>0$. Since $\beta \mapsto h_{c}^{\text {que }}(\beta)$ is convex, it must therefore be strictly increasing (see Fig. 1).

4. Disorder has a tendency to smoothen the phase transition. In Caravenna and den Hollander [14] a general smoothing inequality is derived that reads as follows:

- For every $\beta>0$ there exist $C(\beta)<\infty$ and $\delta(\beta)>0$ such that

$$
0 \leq \mathrm{F}^{\text {que }}\left(\beta, h_{c}^{\text {que }}(\beta)-\delta\right) \leq C(\beta) \delta^{2} \quad \forall 0 \leq \delta \leq \delta(\beta),
$$

i.e., the quenched phase transition is at least of second order.

Unfortunately, the key assumption under which this smoothing inequality is derived is not obviously met by spatially extended pinning: it does when the tail exponent of $\varphi$ (recall (1.44)) satisfies $\vartheta \in(2, \infty)$, but it is unclear whether it also does when $\vartheta \in(0,2]$. Indeed, the key assumption in [14] requires that $Z_{N, \beta, h}^{\omega, \mathrm{c}} \geq N^{-\gamma} c_{\beta, h}^{\omega}$ for some $\gamma>0$ with $\mathbb{E}\left[\log c_{\beta, h}^{\omega}\right]>-\infty$. Applying Jensen's inequality to (1.12), we get

$$
Z_{N, \beta, h}^{\omega, \mathrm{c}} \geq \mathrm{e}^{\sum_{n=1}^{N}\left(\beta \omega_{n}-h\right) \mathrm{E}\left[\varphi\left(S_{n}\right) \mid S_{N}=0\right]} \mathrm{P}\left(S_{N}=0\right), \quad N \in \mathbb{N} .
$$

In general, if $\mathrm{P}\left(S_{N}=0\right) \geq C N^{-\gamma}$ for some $\gamma, C>0$ and all $N \in \mathbb{N}$ and, furthermore, $\sup _{N \in \mathbb{N}} \sum_{n=1}^{N}\left|\mathrm{E}\left[\varphi\left(S_{n}\right) \mid S_{N}=0\right]\right|<\infty$, then the assumption is met (recall (1.3)). For the Bessel random walk, the former holds by (1.38) for $\gamma=1-\alpha$, while the latter holds by (1.41) when $\varphi(x) \sim c|x|^{-\vartheta}$ with $\vartheta \in(2, \infty)$, 
because in that case, for $n \leq N / 2$ (by symmetry), $\sup _{N \in \mathbb{N}} \mathrm{E}\left[\varphi\left(S_{n}\right) \mid S_{N}=0\right] \lesssim$ $\varphi(\sqrt{n}) \asymp n^{-\vartheta / 2}$ is summable.

5. Theorems 1.6-1.8 give detailed information about the scaling of the annealed free energy and the annealed critical curve in the limit of weak interaction. The scaling limits correspond to annealed free energies and annealed critical curves for Brownian versions of the model involving the Bessel process $X^{\alpha}$, which are interesting in their own right. The result is only valid for the Bessel random walk, and shows a trichotomy depending on the parameters $\alpha$ and $\vartheta$.

- The regime $\vartheta \in(0,1-\alpha)$ corresponds to a long-range interaction potential and is not pinning-like. When localized, the continuum polymer spends a positive fraction of the time near any height $x \in \mathbb{R}$, and this fraction tends to zero as $|x| \downarrow 0$ or $|x| \rightarrow \infty$. Away from 0 it does not behave like the Bessel process conditioned to return to 0 .

- The regime $\vartheta \in(1-\alpha, 2(1-\alpha))$ corresponds to an intermediate-range interaction potential and exhibits some pinning-like features. When localized, the continuum polymer visits 0 a positive fraction of the time. Away from 0 it does not behave like the Bessel process conditioned to return to 0 .

- The regime $\vartheta \in(2(1-\alpha), \infty)$ corresponds to a short-range interaction potential and is pinning-like. When localized, the continuum polymer visits 0 a positive fraction of the time. Away from 0 it behaves like the Bessel process conditioned to return to 0 .

In the last regime the behaviour is similar to that of the homogeneous pinning model with $\varphi(x)=c 1_{\{x=0\}}$, for which it is known that $h_{c}^{\text {ann }}(\beta) \sim \frac{1}{2} c \beta^{2}, \beta \downarrow 0$ (see Giacomin [21], [22], den Hollander [24]). In fact, the proof of Theorem 1.8 will show that the scaling in the last regime is valid for any $\varphi$ such that $c^{*}\left[\varphi^{2}\right]$ and $c^{*}[\varphi]$ are finite, i.e., (1.44) may be replaced by the weaker condition $\varphi(x)=$ $O\left(|x|^{-2(1-\alpha)-\varepsilon}\right)$ for some $\varepsilon>0$.

6. The three regimes for $\vartheta$ represent three universality classes. The critical cases $\vartheta=1-\alpha$ and $\vartheta=2(1-\alpha)$ are more delicate and we have skipped them. Also, we have not investigated what happens when the scaling of the interaction potential in (1.44) is modulated by a slowly varying function. For the same reason we have assumed that the error term in (1.8) is $O\left(|x|^{-(1+\varepsilon)}\right)$ with $\varepsilon>0$ rather than $o\left(|x|^{-1}\right)$, since the latter may give rise to modulation by slowly varying functions in (1.38) and (1.39) (see Alexander [2]).

7. Theorems 1.6-1.8 are deduced from scaling properties of the annealed partition function. They are not specific to the annealed model. In fact, analogous results hold for every homogeneous pinning potential that is a linear combination of potentials with a polynomial tail. See Sect. 4 for more details.

8. By the considerations made in Sect. 3 , the annealed model defined in (1.20)-(1.21) is localized (i.e., $\left.\mathrm{F}^{\text {ann }}(\beta, h)>0, h<h_{c}^{\text {ann }}(\beta)\right)$ if and only if

$$
\sum_{m \in \mathbb{N}} \mathrm{E}\left[\mathrm{e}^{\sum_{n=1}^{m} \psi_{\beta, h}\left(S_{n}\right)} 1_{\left\{\tau_{1}=m\right\}}\right]>1,
$$


where we recall that $\tau_{1}$ denotes the first return time of $S$ to 0 . Although the starting point 0 seems to play a special role in (1.62), it can be shown that the criterion in (1.62) is invariant under spatial shifts of $\psi_{\beta, h}$ (see Appendix C).

A natural question is what happens when the random walk $S$ is transient, i.e., $\mathrm{P}\left(\tau_{1}<\right.$ $\infty)=\sum_{n \in \mathbb{N}} K(n)=: r<1$. For the constrained partition function $Z_{N, \beta, h}^{\omega, \mathrm{c}}$, working with a transient renewal process with law $K$ is equivalent to working with a recurrent renewal process with law $K / r$ and adding a depinning term $\sum_{n=1}^{N}(\log r) 1_{\left\{S_{n}=0\right\}}$ in the exponential in (1.12). This amounts to replacing $\psi_{\beta, h}(x)$ by $\psi_{\beta, h}(x)+(\log r) 1_{\{x=0\}}$, and so instead of (1.62) the localization condition for the annealed model becomes

$$
\sum_{m \in \mathbb{N}} \mathrm{E}\left[\mathrm{e}^{\sum_{n=1}^{m} \psi_{\beta, h}\left(S_{n}\right)} 1_{\left\{\tau_{1}=m\right\}}\right]>\frac{1}{r}
$$

(I) For the copolymer model we have $\varphi(x)=\varphi^{\mathrm{cop}}(x)=1_{\{x \leq 0\}}$ (recall (1.10)) and $\psi_{\beta, h}(x)=\left(\frac{1}{2} \beta^{2}-h\right) 1_{(-\infty, 0]}(x)$. Therefore $h_{c}^{\text {ann }}(\beta)=\frac{1}{2} \beta^{2}$ and, in fact, the left-hand side of (1.62) is $\leq 1$ for $h \leq h_{c}^{\mathrm{ann}}(\beta)$ and is $=\infty$ for $h>h_{c}^{\mathrm{ann}}(\beta)$. This means that the annealed critical curve $h_{c}^{\text {ann }}$ does not depend on $r$, and hence neither do the bounds in (1.43). In other words, making the underlying renewal process transient or, equivalently, adding a homogeneous depinning term at zero, does not modify the annealed critical curve of the copolymer model. In essence this is due to the fact that the copolymer potential is long range (i.e., $\psi_{\beta, h}(x)$ does not vanish as $x \rightarrow-\infty)$.

(II) For the pinning model, adding a depinning term at zero amounts to shifting $h$ and this may have an effect. In essence this is due to the fact that the pinning potential $\varphi(x)=\varphi^{\mathrm{pin}}(x)=1_{\{x=0\}}$ is short range.

\subsection{Open problems and outline}

1. Are the inequalities in (1.43) strict for all $\beta>0$ ? For the copolymer model $(\varphi(x)=$ $\left.1_{\{x \leq 0\}}\right)$ the answer is yes for all $\alpha>0$ (Bolthausen et al. [11]). Moreover, it is known that (Bolthausen and den Hollander [10], Caravenna and Giacomin [12])

$$
\lim _{\beta \downarrow 0} \beta^{-1} h_{c}^{\text {ann }}(\beta)=1, \quad \lim _{\beta \downarrow 0} \beta^{-1} h_{c}^{\text {que }}(\beta)=C(\alpha),
$$

with $C(\alpha) \in(1 /(1+\alpha), 1)$ for $0<\alpha<1$ (Bolthausen et al. [11]) and $C(\alpha)=$ $1 /(1+\alpha)$ for $\alpha \geq 1$ (Berger et al. [4]). For the pinning model $\left(\varphi(x)=1_{\{x=0\}}\right)$ the answer depends on $\alpha$ : no for $0<\alpha<\frac{1}{2}$ (the upper bound is an equality), yes for $\alpha>\frac{1}{2}$. Moreover, it is known that

$$
\lim _{\beta \downarrow 0} \beta^{-2} h_{c}^{\text {ann }}(\beta)=\frac{1}{2}, \quad \lim _{\beta \downarrow 0} \beta^{-2} h_{c}^{\text {que }}(\beta)=C^{\prime}(\alpha),
$$

with $C^{\prime}(\alpha)=\frac{1}{2}$ for $0<\alpha<1$ and $C^{\prime}(\alpha) \in\left(0, \frac{1}{2}\right)$ for $\alpha>1$. As a matter of fact, refined estimates are available: 
- $\alpha \in\left(0, \frac{1}{2}\right): h_{c}^{\text {ann }}(\beta)=h_{c}^{\text {que }}(\beta)$ for $\beta>0$ small enough (Alexander [1], see also Cheliotis and den Hollander [17]);

- $\alpha \in\left(\frac{1}{2}, 1\right): h_{c}^{\text {ann }}(\beta)-h_{c}^{\text {que }}(\beta) \sim c_{\alpha} \beta^{2 \alpha /(2 \alpha-1)}$ as $\beta \downarrow 0$ for a universal constant $c_{\alpha} \in(0, \infty)$ (Caravenna et al. [16], and previously Alexander and Zygouras [3], Derrida et al. [20]).

- $\alpha=\frac{1}{2}: h_{c}^{\text {ann }}(\beta)-h_{c}^{\text {que }}(\beta)=\exp \left(-\frac{\pi}{2 \beta^{2}}[1+o(1)]\right)$ (Berger and Lacoin [6], and previously Giacomin et al. [23]).

For an overview, we refer the reader to Giacomin [22].

2. Determine the order of the quenched phase transition. For the copolymer model it is known that the phase transition is of infinite order when $\alpha=0$ (Berger et al. [5]). The same is conjectured to be true for $\alpha \in(0,1)$.

3 . Identify the scaling for weak interaction of the annealed model in the critical cases $\vartheta=1-\alpha$ and $\vartheta=2(1-\alpha)$.

4. Identify the scaling for weak interaction of the quenched model. Because of Theorem 1.5, the same exponent $E$ as in (1.54) applies.

5. The qualitative shape of the critical curve in Fig. 1 depends on our assumption in (1.1) that $\varphi \geq 0$. A reflected picture holds when $\varphi \leq 0$. It appears that for $\varphi$ with mixed signs there are two critical curves $\beta \mapsto h_{c, 1}^{\text {que }}(\beta)$ and $\beta \mapsto h_{c, 2}^{\text {que }}(\beta)$, separating a single quenched delocalized phase $\mathcal{D}^{\text {que }}$ from two quenched localized phases $\mathcal{L}_{1}^{\text {que }}$ and $\mathcal{L}_{2}^{\text {que }}$ that lie above $\mathcal{D}^{\text {que }}$, respectively, below $\mathcal{D}^{\text {que }}$. What are the properties of these critical curves?

6. What happens when $\beta=\beta_{N}$ and $h=h_{N}$ with $\beta_{N}, h_{N} \downarrow 0$ as $N \rightarrow \infty$.

7. Is it possible to include non-nearest-neighbour random walks?

Outline The remainder of this paper is organized as follows. Theorem 1.4 is proved in Sect. 2, Theorem 1.5 in Sect. 3 and Theorems 1.6-1.8 in Sect. 4. "Appendices A and B" collect a few technical facts that are needed along the way.

\section{Proof of Theorem 1.4}

In Sect. 2.1 we formulate annealed and quenched large deviation principles (LDPs) that are an adaptation to our model of the LDPs developed in Birkner [7] and Birkner et al. [8]. The latter concern LDPs for random sequences of words cut out from random sequences of letters according to a renewal process. In Sect. 2.2 we formulate variational characterizations of the annealed and quenched critical curves that are an adaptation of the characterizations derived for the pinning model in Cheliotis and den Hollander [17] and for the copolymer model in Bolthausen et al. [11]. In Sect. 2.3 we explain how the variational characterizations follow from the LDPs via Varadhan's lemma.

\subsection{Annealed and quenched LDP}

Our starting observation is that the partition function in (1.4) depends on the sequence of words $Y=\left(Y_{i}\right)_{i \in \mathbb{N}}$ determined by the disorder and by the excursions of the polymer, 
namely,

$$
Y_{i}=Y_{i}(\omega, S):=\left(\left(\omega_{\tau_{i-1}+1}, \ldots, \omega_{\tau_{i}}\right),\left(S_{\tau_{i-1}+1}, \ldots, S_{\tau_{i}}\right)\right), \quad i \in \mathbb{N}
$$

where $\tau=\left(\tau_{i}\right)_{i \in \mathbb{N}}$ is the sequence of epochs of the successive visits of the polymer to zero $\left(\tau_{0}=0\right)$. Note that the random variables $Y_{i}$ take their values in the space $\tilde{\Gamma}:=\bigcup_{n \in \mathbb{N}} \Gamma^{n}$ with $\Gamma:=\mathbb{R} \times \mathbb{Z}$.

To capture the role of $Y$, we introduce its empirical process,

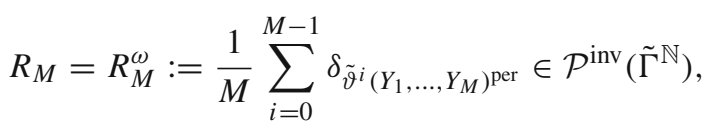

where $\mathcal{P}^{\text {inv }}\left(\tilde{\Gamma}^{\mathbb{N}}\right)$ denotes the set of probability measures on $\tilde{\Gamma}^{\mathbb{N}}$ that are invariant under the left-shift $\tilde{\vartheta}$ acting on $\tilde{\Gamma}^{\mathbb{N}}$. The superscript $\omega$ reminds us that the random variables $Y_{i}$ are functions of $\omega$. We must average over $S$ while keeping $\omega$ fixed. Note that, under the annealed law $\mathbb{P} \otimes \mathrm{P}, Y$ is i.i.d. with the following marginal law $q_{0}$ on $\tilde{\Gamma}$ :

$$
\begin{aligned}
& q_{0}\left(\left(\mathrm{~d} x_{1}, \ldots, \mathrm{d} x_{n}\right) \times\left\{\left(s_{1}, \ldots, s_{n}\right)\right\}\right) \\
& \quad:=(\mathbb{P} \otimes \mathrm{P})\left(Y_{1} \in\left(\mathrm{d} x_{1}, \ldots, \mathrm{d} x_{n}\right) \times\left\{\left(s_{1}, \ldots, s_{n}\right)\right\}\right) \\
& \quad=K(n) v\left(\mathrm{~d} x_{1}\right) \cdots v\left(\mathrm{~d} x_{n}\right) \mathrm{P}\left(\left(S_{1}, \ldots, S_{n}\right)=\left(s_{1}, \ldots, s_{n}\right) \mid \tau_{1}=n\right), \\
& n \in \mathbb{N}, x_{1}, \ldots, x_{n} \in \mathbb{R}, s_{1}, \ldots, s_{n} \in \mathbb{Z},
\end{aligned}
$$

where $K(n):=\mathrm{P}\left(\tau_{1}=n\right)$ and $v(\mathrm{~d} x):=\mathbb{P}\left(\omega_{1} \in \mathrm{d} x\right)$.

The specific relative entropy of $Q$ w.r.t. $q_{0}^{\otimes \mathbb{N}}$ is defined by

$$
H\left(Q \mid q_{0}^{\otimes \mathbb{N}}\right):=\lim _{N \rightarrow \infty} \frac{1}{N} h\left(\tilde{\pi}_{N} Q \mid q_{0}^{N}\right)
$$

where $\tilde{\pi}_{N} Q \in \mathcal{P}\left(\tilde{\Gamma}^{N}\right)$ denotes the projection of $Q$ onto the first $N$ words, $h(\cdot \mid \cdot)$ denotes relative entropy, and the limit is non-decreasing. The following annealed LDP is standard (see Dembo and Zeitouni [19, Section 6.5]).

Proposition 2.1 (Annealed LDP). The family $(\mathbb{P} \otimes \mathrm{P})\left(R_{M}^{\omega} \in \cdot\right), M \in \mathbb{N}$, satisfies the LDP on $\mathcal{P}^{\text {inv }}\left(\tilde{\Gamma}^{\mathbb{N}}\right)$ with rate $M$ and with rate function $I^{\mathrm{ann}}$ given by

$$
I^{\text {ann }}(Q):=H\left(Q \mid q_{0}^{\otimes \mathbb{N}}\right), \quad Q \in \mathcal{P}^{\text {inv }}\left(\tilde{\Gamma}^{\mathbb{N}}\right) .
$$

This rate function is lower semi-continuous, has compact level sets, has a unique zero at $q_{0}^{\otimes \mathbb{N}}$, and is affine.

The quenched LDP is more delicate and requires extra notation. The reverse operation of cutting words out of a sequence of letters is glueing words together into a sequence of letters. Formally, this is done by defining a concatenation map $\kappa$ from $\tilde{\Gamma}^{\mathbb{N}}$ 
to $\Gamma^{\mathbb{N}}$. This map induces in a natural way a map from $\mathcal{P}\left(\tilde{\Gamma}^{\mathbb{N}}\right)$ to $\mathcal{P}\left(\Gamma^{\mathbb{N}}\right)$, the sets of probability measures on $\tilde{\Gamma}^{\mathbb{N}}$ and $\Gamma^{\mathbb{N}}$ (endowed with the topology of weak convergence). The concatenation $q_{0}^{\otimes \mathbb{N}} \circ \kappa^{-1}$ of $q_{0}^{\otimes \mathbb{N}}$ equals $v^{\otimes \mathbb{N}}$, as is evident from (2.3).

For $Q \in \mathcal{P}^{\text {inv }}\left(\tilde{\Gamma}^{\mathbb{N}}\right)$, let $m_{Q}:=E_{Q}\left(\tau_{1}\right) \in[1, \infty]$ be the average word length under $Q$ ( $E_{Q}$ denotes expectation under the law $Q$ and $\tau_{1}$ is the length of the first word). Let

$$
\mathcal{P}^{\text {inv,fin }}\left(\tilde{\Gamma}^{\mathbb{N}}\right):=\left\{Q \in \mathcal{P}^{\text {inv }}\left(\tilde{\Gamma}^{\mathbb{N}}\right): m_{Q}<\infty\right\}
$$

For $Q \in \mathcal{P}^{\text {inv, fin }}\left(\tilde{\Gamma}^{\mathbb{N}}\right)$, define

$$
\Psi_{Q}:=\frac{1}{m_{Q}} E_{Q}\left[\sum_{k=0}^{\tau_{1}-1} \delta_{\vartheta^{k} \kappa(Y)}\right] \in \mathcal{P}^{\mathrm{inv}}\left(\Gamma^{\mathbb{N}}\right)
$$

Think of $\Psi_{Q}$ as the shift-invariant version of $Q \circ \kappa^{-1}$ obtained after randomizing the location of the origin. This randomization is necessary because a shift-invariant $Q$ in general does not give rise to a shift-invariant $Q \circ \kappa^{-1}$.

The following quenched LDP is a straight adaptation of the one derived in Birkner, Greven and den Hollander [8].

Proposition 2.2 (Quenched LDP). For $\mathbb{P}$-a.e. $\omega$ the family $\mathrm{P}\left(R_{M}^{\omega} \in \cdot\right), M \in \mathbb{N}$, satisfies the LDP on $\mathcal{P}^{\text {inv }}\left(\tilde{\Gamma}^{\mathbb{N}}\right)$ with rate $M$ and with rate function given by

$$
I^{\text {que }}(Q):= \begin{cases}H\left(Q \mid q_{0}^{\otimes \mathbb{N}}\right)+\alpha m_{Q} H\left(\Psi_{Q} \mid v^{\otimes \mathbb{N}}\right), & Q \in \mathcal{P}^{\text {inv,fin }}\left(\tilde{\Gamma}^{\mathbb{N}}\right), \\ \lim _{\delta \downarrow 0} \inf _{Q^{\prime} \in B_{\delta}(Q) \cap \mathcal{P}^{\text {inv, fin }}\left(\tilde{\Gamma}^{\mathbb{N}}\right)} I^{\text {que }}\left(Q^{\prime}\right), \quad Q \in \mathcal{P}^{\text {inv }}\left(\tilde{\Gamma}^{\mathbb{N}}\right) \backslash \mathcal{P}^{\text {inv }, \text { fin }}\left(\tilde{\Gamma}^{\mathbb{N}}\right),\end{cases}
$$

where $\alpha$ is the exponent in (1.6) and $B_{\delta}(Q)$ is the $\delta$-ball around $Q$ (in any appropriate metric). This rate function is lower semi-continuous, has compact level sets, has a unique zero at $q_{0}^{\otimes \mathbb{N}}$, and is affine.

Remark 2.3 In [8] a formula was claimed for $I^{\text {que }}$ on $\mathcal{P}^{\text {inv }}\left(\tilde{\Gamma}^{\mathbb{N}}\right) \backslash \mathcal{P}^{\text {inv, fin }}\left(\tilde{\Gamma}^{\mathbb{N}}\right)$ based on a truncation approximation for the average word length. As pointed out by JeanChristophe Mourrat (private communication), the proof of this formula in [8] is flawed. The formula itself may still be correct, but no proof is currently available. In the present paper we will only need to know $I^{\text {que }}$ on $\mathcal{P}^{\text {inv, fin }}\left(\tilde{\Gamma}^{\mathbb{N}}\right)$.

\subsection{Variational criterion for localization}

For $N \in \mathbb{N}$, let $\ell_{N}$ be the number of returns to zero of the polymer before epoch $N$, i.e.,

$$
\ell_{N}:=\max \left\{i \in \mathbb{N}_{0}: \tau_{i} \leq N\right\}
$$


Let $\Phi: \tilde{\Gamma} \rightarrow \mathbb{R}$ be defined by

$$
\Phi_{\beta, h}\left(\left(x_{1}, \ldots, x_{n}\right),\left(s_{1}, \ldots, s_{n}\right)\right):=\sum_{m=1}^{n}\left(\beta x_{m}-h\right) \varphi\left(s_{m}\right) .
$$

Then the constrained quenched partition function defined in (1.12) can be written as

$$
Z_{N, \beta, h}^{\omega, \mathrm{c}}=\mathrm{E}\left[\mathrm{e}^{\sum_{i=1}^{\ell_{N}} \Phi_{\beta, h}\left(Y_{i}\right)} 1_{\left\{S_{N}=0\right\}}\right]=\mathrm{E}\left[\mathrm{e}^{\ell_{N} \int_{\tilde{\Gamma}} \Phi_{\beta, h} \mathrm{~d}\left(\tilde{\pi}_{1} \mathbb{R}_{\ell_{N}}^{\omega}\right)} 1_{\left\{S_{N}=0\right\}}\right],
$$

while the constrained annealed partition function defined in (1.23) can be written as

$$
Z_{N, \beta, h}^{\mathrm{ann}, \mathrm{c}}=(\mathbb{E} \otimes \mathrm{E})\left[\mathrm{e}^{\sum_{i=1}^{\ell_{N}} \Phi_{\beta, h}\left(Y_{i}\right)} 1_{\left\{S_{N}=0\right\}}\right]=(\mathbb{E} \otimes \mathrm{E})\left[\mathrm{e}^{\ell_{N} \int_{\tilde{\Gamma}} \Phi_{\beta, h} \mathrm{~d}\left(\tilde{\pi}_{1} \mathbb{R}_{\ell_{N}}^{\omega}\right)} 1_{\left\{S_{N}=0\right\}}\right],
$$

where $\tilde{\pi}_{1} Q$ denotes the projection of $Q$ onto the space $\tilde{\Gamma}$ of the first word.

With the help of Propositions 2.1-2.2 we can derive the following variational characterization of the annealed and the quenched critical curve. Note that $\mathrm{F}^{\mathrm{ann}}(\beta, h)>0$ if and only if $h<h_{c}^{\text {ann }}(\beta)$ and $\mathrm{F}^{\text {que }}(\beta, h)>0$ if and only if $h<h_{c}^{\text {que }}(\beta)$.

Theorem 2.4 [Annealed localization]. For every $\beta, h>0$,

$$
\mathrm{F}^{\mathrm{ann}}(\beta, h)>0 \Longleftrightarrow \sup _{\substack{Q \in \mathcal{P}^{\text {inv }}\left(\tilde{\Gamma}^{\mathbb{N}}\right): \\ m_{Q}<\infty, I^{\text {ann }}(Q)<\infty}}\left\{\int_{\tilde{\Gamma}} \Phi_{\beta, h} d\left(\tilde{\pi}_{1} Q\right)-I^{\mathrm{ann}}(Q)\right\}>0
$$

Theorem 2.5 [Quenched localization]. For every $\beta, h>0$,

$$
\mathrm{F}^{\text {que }}(\beta, h)>0 \Longleftrightarrow \sup _{\substack{Q \in \mathcal{P}^{\text {inv }}\left(\tilde{\Gamma}^{\mathbb{N}}\right): \\ m_{Q}<\infty, I^{\text {ann }}(Q)<\infty}}\left\{\int_{\tilde{\Gamma}} \Phi_{\beta, h} d\left(\tilde{\pi}_{1} Q\right)-I^{\text {que }}(Q)\right\}>0 .
$$

As we will see below, the role of the conditions $m_{Q}<\infty$ and $I^{\text {ann }}(Q)<\infty$ under the two suprema is to ensure that $\int_{\tilde{\Gamma}} \Phi_{\beta, h} \mathrm{~d}\left(\tilde{\pi}_{1} Q\right)<\infty$, so that the suprema are well defined. The condition $m_{Q}<\infty$ under the second supremum allows us to use the representation in (2.8). We will see in Sect. 3 how the variational formulas in (2.13)-(2.14) can be exploited.

\subsection{Proof of Theorems 2.4-2.5}

The proof uses arguments developed in Bolthausen et al. [11]. Theorems 2.4-2.5 follow from Propositions 2.1-2.2 with the help of Varadhan's lemma applied to (2.11)-(2.12). 
The only difficulty we need to deal with is the fact that both $Q \mapsto m_{Q}$ and $Q \mapsto$ $\Phi_{\beta, h}^{*}(Q)=\int_{\tilde{\Gamma}} \Phi_{\beta, h} \mathrm{~d}\left(\tilde{\pi}_{1} Q\right)$ are neither bounded nor continuous in the weak topology. Therefore an approximation argument is required, which is worked out in detail in [11, Appendices A-D] for the case of the copolymer interaction potential in (1.10). This approximation argument shows why the restriction to $m_{Q}<\infty$ and $I^{\text {ann }}(Q)<\infty$ may be imposed, a key ingredient being that $I^{\text {ann }}(Q)<\infty$ implies $\Phi_{\beta, h}^{*}(Q)<\infty$. The proof in [11, Appendices A-D] readily carries over because our condition on the interaction potential in (1.1) reflects the properties of the copolymer interaction potential. We sketch the main line of thought. Throughout the sequel $\beta, h>0$ are fixed.

Proof of Theorem 2.5 Following the argument in [11, Appendix A], we show that

(1) For every $g>0, M \mapsto \Psi_{\beta, h}\left(R_{M}^{\omega}\right)-g m_{R_{M}^{\omega}}$ is bounded $\omega$-a.s.

(2) For every $\varrho \in \mathcal{P}(\mathbb{N}), v \in \mathcal{P}(\mathbb{R})$ and $p=\left(p_{n}\right)_{n \in \mathbb{N}}$ with $p_{n} \in \mathcal{P}\left(\mathbb{Z}^{n}\right)$, there exist $\gamma>0$ and $K=K(\varrho, v, p ; \gamma)>0$ such that $\Phi_{\beta, h}^{*}(Q) \leq \gamma h\left(\tilde{\pi}_{1} Q \mid q_{\varrho, \nu, p}\right)+K$ for all $Q \in \mathcal{P}^{\text {inv }}\left(\tilde{\Gamma}^{\mathbb{N}}\right)$ with $h\left(\tilde{\pi}_{1} Q \mid q_{\varrho, v, p}\right)<\infty$, where (compare with (2.3))

$$
\begin{aligned}
& q_{\varrho, v, p}\left(\left(\mathrm{~d} x_{1}, \ldots, \mathrm{d} x_{n}\right) \times\left\{\left(s_{1}, \ldots, s_{n}\right)\right\}\right)=\varrho(n) v\left(\mathrm{~d} x_{1}\right) \cdots v\left(\mathrm{~d} x_{n}\right) p_{n}\left(s_{1}, \ldots, s_{n}\right), \\
& n \in \mathbb{N}, x_{1}, \ldots, x_{n} \in \mathbb{R}, s_{1}, \ldots, s_{n} \in \mathbb{Z}
\end{aligned}
$$

The proof uses the fact that the conditions in (1.1) allow us to approximate $\varphi$ by a multiple of $\varphi^{\text {cop }}$ (recall (1.10)) uniformly on $\mathbb{Z} \backslash[-L, L]$ at arbitrary precision as $L \rightarrow \infty$. The proof also uses a concentration of measure estimate for the disorder, which is proved in [11, Appendix D].

For $g>0$, define the quenched free energy

$$
\mathrm{F}^{\mathrm{que}}(\beta, h ; g):=\lim _{N \rightarrow \infty} \frac{1}{N} \log Z_{N, \beta, h, g}^{\mathrm{que}}
$$

where

$$
Z_{N, \beta, h, g}^{\text {que }}:=\mathrm{E}\left[\mathrm{e}^{N\left\{\Phi_{\beta, h}^{*}\left(R_{M}^{\omega}\right)-g m_{R_{M}^{\omega}}\right\}}\right]
$$

is the quenched partition function in which every letter gets an energetic penalty $-g$. Following the argument in [11, Appendix B], we use (1) and (2) to show that, for every $g>0$,

$$
\mathrm{F}^{\text {que }}(\beta, h ; g)=\sup _{\substack{Q \in \mathcal{R}: \\ m_{Q}<\infty, I^{\text {ann }}(Q)<\infty}}\left\{\int_{\tilde{\Gamma}^{\mathbb{N}}} \Phi_{\beta, h}^{*}(Q)-g m_{Q}-I^{\text {ann }}(Q)\right\}
$$

where $\mathcal{R}$ is the set of shift-invariant probability measures under which the concatenation of words produces a letter sequence that has the same asymptotic statistics as a 
typical realisation of $Y$, i.e.,

$$
\mathcal{R}:=\left\{Q \in \mathcal{P}^{\text {inv }}\left(\tilde{\Gamma}^{\mathbb{N}}\right): w-\lim _{M \rightarrow \infty} \frac{1}{M} \sum_{k=0}^{M-1} \delta_{\vartheta^{k} \kappa(Y)}=\mu^{\otimes \mathbb{N}} Q-\text { a.s. }\right\}
$$

( $w-$ lim means weak limit). The proof of (2.18) carries over verbatim. What (2.18) says is that Varadhan's lemma applies to (2.16)-(2.17) because of the control enforced by (1) and (2).

Following the argument in [11, Appendix C], we show that

$$
\lim _{g \downarrow 0} \mathrm{~F}^{\text {que }}(\beta, h ; g)=\mathrm{F}^{\text {que }}(\beta, h)
$$

with

$$
\mathrm{F}^{\text {que }}(\beta, h)=\sup _{\substack{\left.Q \in \mathcal{P}^{\text {inv }} \\ m \tilde{\Gamma}^{\mathbb{N}}\right):}}\left\{\int_{\tilde{\Gamma}^{\mathbb{N}}} \Phi_{\beta, h}^{*}(Q)-I^{\text {que }}(Q)\right\}
$$

Here, in the passage from (2.18) to (2.21), the constraint in $\mathcal{R}$ disappears from the variational characterization, while $I^{\text {ann }}(Q)$ is replaced by $I^{\text {que }}(Q)$. The reason is that for every $Q \in \mathcal{P}^{\text {inv }}\left(\tilde{\Gamma}^{\mathbb{N}}\right)$ there exists a sequence $\left(Q_{n}\right)_{n \in \mathbb{N}}$ in $\mathcal{R}$ such that $Q=$ $w-\lim _{n \rightarrow \infty} Q_{n}=Q$ and $\lim _{n \rightarrow \infty} I^{\text {ann }}\left(Q_{n}\right)=I^{\text {que }}(Q)$. The proof of (2.21) carries over verbatim. The supremum in the right-hand side of (2.21) is the same as the supremum in the right-hand side of (2.14).

Proof of Theorem 2.4 Varadhan's lemma also applies to

$$
Z_{N, \beta, h}^{\mathrm{ann}}:=\mathrm{E}\left[\mathrm{e}^{N \Phi_{\beta, h}^{*}\left(R_{M}^{\omega}\right)}\right]
$$

and yields

$$
\mathrm{F}^{\mathrm{ann}}(\beta, h):=\lim _{N \rightarrow \infty} \frac{1}{N} \log Z_{N, \beta, h}^{\mathrm{ann}}
$$

with

$$
\mathrm{F}^{\mathrm{ann}}(\beta, h)=\sup _{\substack{Q \in \mathcal{P}^{\text {inv }}\left(\tilde{\Gamma}^{\mathbb{N}}\right): \\ m Q<\infty, I^{\text {ann }}(Q)<\infty}}\left\{\int_{\tilde{\Gamma}^{\mathbb{N}}} \Phi_{\beta, h}^{*}(Q)-I^{\text {ann }}(Q)\right\}
$$

Again, this works because of the control enforced by (2). The supremum in the righthand side of (2.24) is the same as the supremum in the right-hand side of (2.13). 


\section{Proof of Theorem 1.5}

The upper bound in (1.43) is immediate from Theorems 2.4-2.5 and the inequality $I^{\text {que }} \geq I^{\text {ann }}$ (see also (1.27)). In Sects. 3.1-3.3 we prove the lower bound in (1.43). This lower bound is the analogue of what for the copolymer model is called the Monthus-Bodineau-Giacomin lower bound (see Giacomin [21], den Hollander [24]).

\subsection{A sufficient criterion for quenched localization}

The quenched rate function can be written as

$$
I^{\text {que }}(Q)=(1+\alpha) I^{\text {ann }}(Q)-\alpha R(Q), \quad m_{Q}<\infty,
$$

with

$$
R(Q):=H\left(Q \mid q_{0}^{\otimes \mathbb{N}}\right)-m_{Q} H\left(\Psi_{Q} \mid v^{\otimes \mathbb{N}}\right)
$$

It can be shown that $R(Q) \geq 0$ for all $Q \in \mathcal{P}^{\text {inv }}\left(\tilde{\Gamma}^{\mathbb{N}}\right): R(Q)$ has the meaning of a concatenation entropy (see Birkner et al. [8]). Therefore, dropping this term in (2.14) we obtain the following sufficient criterion for quenched localization:

$$
\mathrm{F}^{\text {que }}(\beta, h)>0 \Longleftarrow \sup _{\substack{Q \in \mathcal{P}^{\text {inv }}\left(\tilde{\Gamma}^{\mathbb{N}}\right): \\ m_{Q}<\infty, I^{\text {ann }}(Q)<\infty}}\left\{\int_{\tilde{\Gamma}} \Phi \mathrm{d}\left(\tilde{\pi}_{1} Q\right)-(1+\alpha) I^{\text {ann }}(Q)\right\}>0
$$

The right-hand side resembles the necessary and sufficient criterion for annealed localization in (2.13), the only difference being the extra factor $1+\alpha$.

\subsection{Reduction}

Among the laws $Q \in \mathcal{P}^{\text {inv }}\left(\tilde{\Gamma}^{\mathbb{N}}\right)$ with a given marginal law $q \in \mathcal{P}(\tilde{\Gamma})$, the product law $Q=q^{\otimes \mathbb{N}}$ is the unique minimizer of the specific relative entropy $H\left(Q \mid q_{0}^{\otimes \mathbb{N}}\right)$. Therefore the right-hand side of (3.3) reduces to

$$
\sup _{\substack{q \in \mathcal{P}^{\text {inv }}(\tilde{\Gamma}): \\ m_{q}<\infty, h\left(q \mid q_{0}\right)<\infty}}\left\{\int_{\tilde{\Gamma}} \Phi \mathrm{d} q-(1+\alpha) h\left(q \mid q_{0}\right)\right\}>0
$$

where $h(\cdot \mid \cdot)$ denotes relative entropy.

We next show that (3.3) reduces to an even simpler criterion. To that end, let $C_{N}:=\bigcup_{n=1}^{N} \Gamma^{n}$ be the subset of words of length at most $N$. Consider the law 
$\hat{q}_{N} \in \mathcal{P}(\tilde{\Gamma})$ defined by

$$
\frac{\mathrm{d} \hat{q}_{N}}{\mathrm{~d} q_{0}}:=\frac{\mathrm{e}^{\frac{1}{1+\alpha} \Phi} 1_{C_{N}}}{\mathcal{N}_{\alpha, N}}
$$

where

$$
\mathcal{N}_{\alpha, N}:=\int_{\tilde{\Gamma}} \mathrm{e}^{\frac{1}{1+\alpha} \Phi} 1_{C_{N}} \mathrm{~d} q_{0}<\infty
$$

is the normalizing constant. The latter is finite because, by (2.10), $\Phi$ restricted to $C_{N}$ is the sum of at most $N$ random variables with finite exponential moments. Note that also

$$
\int_{\tilde{\Gamma}} \Phi \mathrm{e}^{\frac{1}{1+\alpha} \Phi} 1_{C_{N}} \mathrm{~d} q_{0}<\infty
$$

which yields $h\left(\hat{q}_{N} \mid q_{0}\right)<\infty$. Trivially, $m_{\hat{q}_{N}} \leq N<\infty$. Therefore we are allowed to pick $q=\hat{q}_{N}$ in (3.4), so that (3.4) is satisfied when

$$
(1+\alpha) \log \mathcal{N}_{\alpha, N}>0 .
$$

Since $N$ is arbitrary, this in turn is satisfied when

$$
\mathcal{N}_{\alpha}>1 \quad \text { with } \quad \mathcal{N}_{\alpha}:=\sup _{N \in \mathbb{N}} \mathcal{N}_{\alpha, N}=\int_{\tilde{\Gamma}} \mathrm{e}^{\frac{1}{1+\alpha} \Phi} \mathrm{d} q_{0},
$$

where $\mathcal{N}_{\alpha}=\infty$ is allowed. Conversely, if $\mathcal{N}_{\alpha} \leq 1$, then (3.4) is not satisfied. Indeed, as soon as $\mathcal{N}_{\alpha}<\infty$ we may introduce the law $\hat{q} \in \mathcal{P}(\tilde{\Gamma})$ defined by

$$
\frac{\mathrm{d} \hat{q}}{\mathrm{~d} q_{0}}:=\frac{\mathrm{e}^{\frac{1}{1+\alpha} \Phi}}{\mathcal{N}_{\alpha}}
$$

and rewrite $h\left(q \mid q_{0}\right)=h(q \mid \hat{q})-\frac{1}{1+\alpha} \int_{\tilde{\Gamma}} \Phi \mathrm{d} q+\log \mathcal{N}_{\alpha}$, so that

$$
\int_{\tilde{\Gamma}} \Phi \mathrm{d} q-(1+\alpha) h\left(q \mid q_{0}\right)=(1+\alpha) \log \mathcal{N}_{\alpha}-h(q \mid \hat{q}) \leq 0
$$

where the last inequality holds for any $q$ because $\mathcal{N}_{\alpha} \leq 1$, and so (3.4) fails. Thus, (3.3) reduces to

$$
\mathrm{F}^{\text {que }}(\beta, h)>0 \Longleftarrow \mathcal{N}_{\alpha}>1
$$




\subsection{Application}

As we remarked below (3.3), (2.13) resembles (3.3), the only difference being the factor $1+\alpha$ instead of 1 in front of $I^{\text {ann }}(Q)=H\left(Q \mid q_{0}^{\otimes \mathbb{N}}\right)$. Therefore, repeating the above steps and recalling (2.14), we conclude that

$$
\mathrm{F}^{\mathrm{ann}}(\beta, h)>0 \Longleftrightarrow \mathcal{N}_{0}>1 \text {. }
$$

It follows from (2.10), (2.12) and (3.9) that the condition $\mathcal{N}_{\alpha}>1$ is equivalent to

$$
\mathrm{F}^{\mathrm{ann}}\left(\frac{1}{1+\alpha} \beta, \frac{1}{1+\alpha} h\right)>0
$$

i.e., $\frac{1}{1+\alpha} h<h_{c}^{\text {ann }}\left(\frac{1}{1+\alpha} \beta\right)$, which by (3.12) implies $\mathrm{F}^{\text {que }}(\beta, h)>0$, i.e., $h<h_{c}^{\text {que }}(\beta)$. This completes the proof of the lower bound in (1.43).

Recalling (2.3), (2.10) and the function $\psi_{\beta, h}$ defined in (1.21), we may write $\mathcal{N}_{0}$ as

$$
\begin{aligned}
\mathcal{N}_{0}=\int_{\tilde{\Gamma}} \mathrm{e}^{\Phi} \mathrm{d} q_{0} & =\sum_{m \in \mathbb{N}} \mathbb{E}\left[\mathrm{E}\left[\mathrm{e}^{\sum_{n=1}^{m}\left(\beta \omega_{n}-h\right) \varphi\left(S_{n}\right)} 1_{\left\{\tau_{1}=m\right\}}\right]\right] \\
& =\sum_{m \in \mathbb{N}} \mathrm{E}\left[\mathrm{e}^{\sum_{n=1}^{m} \psi_{\beta, h}\left(S_{n}\right)} 1_{\left\{\tau_{1}=m\right\}}\right]
\end{aligned}
$$

We will analyse this expression in Sect. 4 in the weak interaction limit $\beta, h \downarrow 0$ for the Bessel random walk.

Note that for $h=0$ the expression in (3.15) reduces to

$$
\sum_{m \in \mathbb{N}} \mathbb{E}\left[\mathrm{E}\left[\mathrm{e}^{\sum_{n=1}^{m} \beta \omega_{n} \varphi\left(S_{n}\right)} 1_{\left\{\tau_{1}=m\right\}}\right]\right]
$$

By Jensen and the fact that $\varphi \not \equiv 0$, this sum is $>1$ for all $\beta>0$. Hence $F^{\text {ann }}(\beta, 0)>0$ for all $\beta>0$, which settles the claim made at the end of Sect. 1.6.

\section{Proof of Theorems 1.6-1.8}

To prove our scaling results for weak interaction, we will exploit the invariance principle in (1.37). Recall (1.20)-(1.21). Since $\left\|\psi_{\beta, h}\right\|_{\infty}$ tends to zero as $\beta, h \downarrow 0$, we can do a weak coupling expansion in the spirit of Caravenna et al. [15].

The proof is long and technical. In Sect. 4.1 we outline the general strategy, which leads to three tasks. These tasks are carried out in Sects. 4.2-4.4, respectively. 


\subsection{General strategy}

Fix $\hat{\beta}, \hat{h} \in(0, \infty)$ and $\beta_{N}, h_{N}$ such that, as $N \rightarrow \infty$,

$$
\beta_{N} \sim \hat{\beta} \times \begin{cases}N^{-(1-\vartheta) / 2}, & \vartheta \in(0,1-\alpha) \\ N^{-\alpha / 2}, & \vartheta \in(1-\alpha, 2(1-\alpha)) \\ N^{-\alpha / 2}, & \vartheta \in(2(1-\alpha), \infty)\end{cases}
$$

and

$$
h_{N} \sim \hat{h} \times \begin{cases}N^{-(2-\vartheta) / 2}, & \vartheta \in(0,1-\alpha) \\ N^{-(2-\vartheta) / 2}, & \vartheta \in(1-\alpha, 2(1-\alpha)) \\ N^{-\alpha}, & \vartheta \in(2(1-\alpha), \infty)\end{cases}
$$

We will prove that, for any $T \in(0, \infty)$,

$$
\lim _{N \rightarrow \infty} Z_{T N, \beta_{N}, h_{N}}^{\mathrm{ann}}=\hat{Z}_{T, \hat{\beta}, \hat{h}}^{\mathrm{ann}}:=\hat{\mathrm{E}}\left[\exp \left(\int_{0}^{T} \hat{\psi}_{\hat{\beta}, \hat{h}}\left(X_{t}\right) \mathrm{d} t\right)\right]
$$

(for ease of notation we pretend that $T N$ is integer), where we set

$$
\hat{\psi}_{\hat{\beta}, \hat{h}}(x):= \begin{cases}\frac{1}{2} \hat{\beta}^{2} c^{2}|x|^{-2 \vartheta}-\hat{h} c|x|^{-\vartheta}, & \vartheta \in(0,1-\alpha), \\ \frac{1}{2} \hat{\beta}^{2} c^{*}\left[\varphi^{2}\right] \hat{\delta}_{0}(x)-\hat{h} c|x|^{-\vartheta}, & \vartheta \in(1-\alpha, 2(1-\alpha)) \\ \left\{\frac{1}{2} \hat{\beta}^{2} c^{*}\left[\varphi^{2}\right]-\hat{h} c^{*}[\varphi]\right\} \hat{\delta}_{0}(x), & \vartheta \in(2(1-\alpha), \infty) .\end{cases}
$$

We recall that the renormalized Dirac-function $\hat{\delta}_{0}(\cdot)$ is the notation introduced in (1.36) to make the time integral $\int_{0}^{T} \hat{\psi}_{\hat{\beta}, \hat{h}}\left(X_{t}\right) \mathrm{d} t$ in (4.3) well-defined.

Once the convergence in (4.3) is established, Theorems 1.6-1.8 follow. Indeed, recalling the definitions of $\hat{F}^{\text {ann }}$ in (1.47), (1.49) and (1.51), respectively, we can write

$$
\begin{gathered}
\hat{F}^{\mathrm{ann}}(\hat{\beta}, \hat{h})=\lim _{T \rightarrow \infty} \frac{1}{T} \log \hat{Z}_{T, \hat{\beta}, \hat{h}}^{\mathrm{ann}}=\lim _{T \rightarrow \infty} \frac{1}{T} \log \left(\lim _{N \rightarrow \infty} Z_{T N, \beta_{N}, h_{N}}^{\mathrm{ann}}\right) \\
=\lim _{N \rightarrow \infty} N\left(\lim _{T \rightarrow \infty} \frac{1}{T N} \log Z_{T N, \beta_{N}, h_{N}}^{\mathrm{ann}}\right) \\
=\lim _{N \rightarrow \infty} N F^{\mathrm{ann}}\left(\beta_{N}, h_{N}\right),
\end{gathered}
$$

where the interchange of the limits $T \rightarrow \infty$ and $N \rightarrow \infty$ is justified in Appendix A.4.

To prove (4.3), we write $Z_{T N, \beta_{N}, h_{N}}^{\text {ann }}$ as a series, recall (1.20). Since the potential $\varphi$ is assumed to be symmetric, i.e., $\varphi(-x)=\varphi(x)$ for all $x \in \mathbb{Z}$, we can write $\psi_{\beta, h}\left(S_{n}\right)=\psi_{\beta, h}\left(\left|S_{n}\right|\right)$, and hence

$$
Z_{T N, \beta_{N}, h_{N}}^{\mathrm{ann}}=\mathrm{E}\left[\prod_{n=1}^{T N}\left\{1+\left(\mathrm{e}^{\psi_{\beta_{N}, h_{N}}\left(\left|S_{n}\right|\right)}-1\right)\right\}\right]=1+\sum_{k \in \mathbb{N}} C_{T N, k}
$$


with

$$
C_{T N, k}:=\sum_{1 \leq n_{1}<\cdots<n_{k} \leq T N} \mathrm{E}\left[\prod_{\ell=1}^{k} \chi_{\beta_{N}, h_{N}}\left(\left|S_{n_{\ell}}\right|\right)\right], \quad \chi_{\beta, h}(x):=e^{\psi_{\beta, h}(x)}-1 .
$$

We can write a similar decomposition for $\hat{Z}_{T, \hat{\beta}, \hat{h}}^{\text {ann }}$, namely,

$$
\hat{Z}_{T, \hat{\beta}, \hat{h}}^{\mathrm{ann}}=1+\sum_{k \in \mathbb{N}} \hat{C}_{T, k}
$$

with

$$
\hat{C}_{T, k}:=\frac{1}{k !} \hat{\mathrm{E}}\left[\left(\int_{0}^{T} \mathrm{~d} t \hat{\psi}_{\hat{\beta}, \hat{h}}\left(X_{t}\right)\right)^{k}\right] .
$$

Formally, if we rewrite the $k$-th power of the integral as a $k$-fold integral and afterwards switch the integral and the expectation, then we get the expression

$$
\hat{C}_{T, k}=\int_{\substack{0<t_{1}<\cdots<t_{k}<T \\ x_{1}, \ldots, x_{k} \in[0, \infty)}} \prod_{\ell=1}^{k}\left\{g_{t_{\ell}-t_{\ell-1}}\left(x_{\ell-1}, x_{\ell}\right) \hat{\psi}_{\hat{\beta}, \hat{h}}\left(x_{\ell}\right)\right\} \mathrm{d} t_{1} \cdots \mathrm{d} t_{k} \mathrm{~d} x_{1} \cdots \mathrm{d} x_{k}
$$

with $t_{0}:=x_{0}:=0$. This is justified by Fubini's theorem in the first regime in (4.4), because $\hat{\psi}_{\hat{\beta}, \hat{h}}(x)$ is a genuine function (see (4.4)). However, the second and third regime in (4.4) are more delicate because $\hat{\psi}_{\hat{\beta}, \hat{h}}(x)$ contains the formal term $\hat{\delta}_{0}(x)$. We claim that (4.10) holds in these regimes as well, provided we use the interpretation

$$
" g_{t_{\ell}-t_{\ell-1}}\left(x_{\ell-1}, x_{\ell}\right) \hat{\delta}_{0}\left(x_{\ell}\right) \mathrm{d} x_{\ell} ":=\hat{g}_{t_{\ell}-t_{\ell-1}}\left(x_{\ell-1}, 0\right) \delta_{0}\left(\mathrm{~d} x_{\ell}\right),
$$

where $\hat{g}$ is the function defined in (1.34) and $\delta_{0}$ is the usual Dirac measure at zero, both of which are proper. We prove (4.10) in Sect. 4.2 below.

Remark 4.1 In the third regime, both terms in $\hat{\psi}_{\hat{\beta}, \hat{h}}(x)$ contain $\hat{\delta}_{0}(x)$ (see (4.4)). Therefore (4.10) can be simplified, by the recipe in (4.11), to give

$$
\begin{aligned}
\hat{C}_{T, k} & =\left\{\frac{1}{2} \hat{\beta}^{2} c^{*}\left[\varphi^{2}\right]-\hat{h} c^{*}[\varphi]\right\}^{k} \int_{0<t_{1}<\cdots<t_{k}<T} \prod_{\ell=1}^{k}\left\{\hat{g}_{t_{\ell}-t_{\ell-1}}(0,0)\right\} \mathrm{d} t_{1} \cdots \mathrm{d} t_{k} \\
& =\left\{\frac{1}{2} \hat{\beta}^{2} c^{*}\left[\varphi^{2}\right]-\hat{h} c^{*}[\varphi]\right\}^{k} \int_{0<t_{1}<\cdots<t_{k}<T} \prod_{\ell=1}^{k} \frac{1}{\left(t_{\ell}-t_{\ell-1}\right)^{1-\alpha}} \mathrm{d} t_{1} \cdots \mathrm{d} t_{k} \\
& =\frac{\left[\left\{\frac{1}{2} \hat{\beta}^{2} c^{*}\left[\varphi^{2}\right]-\hat{h} c^{*}[\varphi]\right\} T^{\alpha} \Gamma(\alpha)\right]^{k}}{\Gamma(\alpha k)},
\end{aligned}
$$


where the last equality can be seen to hold by the normalisation constant of the Dirichlet distribution (see also (B.9)). Therefore (4.10) is delicate only in the second regime.

We will prove in Sect. 4.3 that the series in (4.6) and (4.8) have negligible tails:

$$
\begin{gathered}
\forall T \in(0, \infty) \forall \varepsilon \in(0,1) \exists \bar{K}=\bar{K}(T, \varepsilon)<\infty: \\
\quad \limsup _{N \rightarrow \infty} \sum_{k>\bar{K}} C_{T N, k}<\varepsilon, \quad \sum_{k>\bar{K}} \hat{C}_{T, k}<\varepsilon .
\end{gathered}
$$

We will show in Sect. 4.4 that

$$
\forall k \in \mathbb{N}: \quad \lim _{N \rightarrow \infty} C_{T N, k}=\hat{C}_{T, k}
$$

The last two equations combine to yield (4.3) and complete the proof.

\subsection{Proof of (4.10)}

For $\varepsilon>0$ we define a genuine function $\hat{\delta}_{0}^{\varepsilon}(x)$ that is meant to approximate $\hat{\delta}_{0}(x)$ as $\varepsilon \downarrow 0$ (recall (1.35)-(1.36)):

$$
\hat{\delta}_{0}^{\varepsilon}(x):=\frac{c_{\alpha}}{\varepsilon^{2(1-\alpha)}} 1_{(0, \varepsilon)}(x)
$$

We also define an approximate version $\hat{\psi}^{\varepsilon}(x)$ of $\hat{\psi}(x)$ in (4.4) by setting (suppressing the dependence on $\hat{\beta}, \hat{h}$ )

$$
\hat{\psi}^{\varepsilon}(x):=\text { the expression obtained from(4.4)after replacing } \hat{\delta}_{0}(x) \text { by } \hat{\delta}_{0}^{\varepsilon}(x),
$$

so that

$$
\lim _{\varepsilon \downarrow 0} \int_{0}^{T} \hat{\psi}^{\varepsilon}\left(X_{t}\right) \mathrm{d} t=\int_{0}^{T} \hat{\psi}\left(X_{t}\right) \mathrm{d} t \quad \text { in probability. }
$$

Below we prove that this convergence also holds in $L^{k}, k \in \mathbb{N}$. Then, via (4.9), we can write

$$
\begin{aligned}
\hat{C}_{T, k} & =\frac{1}{k !} \lim _{\varepsilon \downarrow 0} \hat{\mathrm{E}}\left[\left(\int_{0}^{T} \mathrm{~d} t \hat{\psi}^{\varepsilon}\left(X_{t}\right)\right)^{k}\right] \\
& =\lim _{\varepsilon \downarrow 0} \int_{\substack{0<t_{1}<\cdots<t_{k}<T \\
x_{1}, \ldots, x_{k} \in[0, \infty)}} \prod_{\ell=1}^{k}\left\{g_{t_{\ell}-t_{\ell-1}}\left(x_{\ell-1}, x_{\ell}\right) \hat{\psi^{\varepsilon}}\left(x_{\ell}\right)\right\} \mathrm{d} t_{1} \cdots \mathrm{d} t_{k} \mathrm{~d} x_{1} \cdots \mathrm{d} x_{k},
\end{aligned}
$$


which coincides precisely with our target Eq. (4.10) with the recipe in (4.11) via the characterization of $\hat{g}$ in (1.33)-(1.34) (note that $x \mapsto g_{t}(x, y)$ is continuous on $[0, \infty)$ ).

To prove that (4.17) holds in $L^{k}, k \in \mathbb{N}$, it is enough to show that all moments of $\int_{0}^{T} \hat{\psi}^{\varepsilon}\left(X_{t}\right) \mathrm{d}$ t are uniformly bounded:

$$
\forall n \in \mathbb{N}: \quad \sup _{\varepsilon \in(0,1)} \hat{\mathrm{E}}\left[\left(\int_{0}^{T} \hat{\psi^{\varepsilon}}\left(X_{t}\right) \mathrm{d} t\right)^{n}\right]<\infty
$$

Since $\hat{\psi}^{\varepsilon}(x)$ is a genuine function, Fubini's theorem tells us that, for every $\varepsilon>0$,

$$
\frac{1}{n !} \hat{\mathrm{E}}_{0}\left[\left(\int_{0}^{T} \hat{\psi}^{\varepsilon}\left(X_{t}\right) \mathrm{d} t\right)^{n}\right]=\int_{0<t_{1}<\cdots<t_{n}<T} \hat{\mathrm{E}}_{0}\left[\prod_{\ell=1}^{n} \hat{\psi}^{\varepsilon}\left(X_{t_{\ell}}\right)\right] \mathrm{d} t_{1} \cdots \mathrm{d} t_{n}
$$

Combining (4.4) abd (4.15)-(4.16), we can bound $\left|\hat{\psi}^{\varepsilon}(x)\right| \leq \bar{\psi}^{\varepsilon}(x):=C_{1} \hat{\delta}_{0}^{\varepsilon}(x)+$ $C_{2}|x|^{-\gamma}$ for suitable constants $C_{1}, C_{2}$ and $\gamma \in\{\vartheta, 2 \vartheta\}<2(1-\alpha)$. Since $\bar{\psi}^{\varepsilon}$ is decreasing, and since $\hat{\mathrm{P}}_{0}\left(X_{t} \in \cdot\right)$ is stochastically dominated by $\hat{\mathrm{P}}_{x}\left(X_{t} \in \cdot\right)$ for any $x \geq 0$, we can bound

$$
\begin{aligned}
& \frac{1}{n !} \hat{\mathrm{E}}_{0}\left[\left(\int_{0}^{T} \hat{\psi}^{\varepsilon}\left(X_{t}\right) \mathrm{d} t\right)^{n}\right] \leq \int_{0<t_{1}<\cdots<t_{n}<T} \prod_{\ell=1}^{n} \hat{\mathrm{E}}_{0}\left[\bar{\psi}^{\varepsilon}\left(X_{t_{\ell}-t_{\ell-1}}\right)\right] \mathrm{d} t_{1} \cdots \mathrm{d} t_{n} \\
& =\int_{0<t_{1}<\cdots<t_{n}<T} \prod_{\ell=1}^{n}\left\{C_{1} \frac{c_{\alpha}}{\varepsilon^{2(1-\alpha)}} \hat{\mathrm{P}}_{0}\left(X_{t_{\ell}-t_{\ell-1}}<\varepsilon\right)+C_{2} \hat{\mathrm{E}}_{0}\left[\left|X_{t_{\ell}-t_{\ell-1}}\right|^{-\gamma}\right]\right\} \\
& \quad \mathrm{d} t_{1} \cdots \mathrm{d} t_{n} \\
& \leq \int_{0<t_{1}<\cdots<t_{n}<T} \prod_{\ell=1}^{n}\left\{\frac{C_{1}}{\left(t_{\ell}-t_{\ell-1}\right)^{1-\alpha}}+\frac{C_{2}^{\prime}}{\left(t_{\ell}-t_{\ell-1}\right)^{\gamma / 2}}\right\} \mathrm{d} t_{1} \cdots \mathrm{d} t_{n},
\end{aligned}
$$

where the last inequality holds by (1.31)-(1.32), for a suitable (and explicit) $C_{2}^{\prime}<\infty$ (note that $\hat{\mathrm{E}}_{0}\left[\left|X_{t_{\ell}-t_{\ell-1}}\right|^{-\gamma}\right]<\infty$ because $\gamma<2(1-\alpha)$ ). The last expression is a finite constant, and so we have completed the proof of (4.10). 


\subsection{Proof of (4.13)}

The second inequality in (4.13) follows from the bounds in (4.18)-(4.21), namely, for some constant $C=C(T)<\infty($ recall that $\gamma<2(1-\alpha))$

$$
\begin{aligned}
& \forall T \in(0, \infty) \quad \forall k \in \mathbb{N}: \\
& \hat{C}_{T, k} \leq \int_{0<t_{1}<\cdots<t_{k}<T}\left\{\prod_{\ell=1}^{k} \frac{C}{\left(t_{\ell}-t_{\ell-1}\right)^{1-\alpha}}\right\} \mathrm{d} t_{1} \cdots \mathrm{d} t_{k}=\frac{\left(C T^{\alpha} \Gamma(\alpha)\right)^{k}}{\Gamma(\alpha k)}
\end{aligned}
$$

(see also Appendix B.1).

To prove the first inequality in (4.13), we recall that, by (4.7),

$$
C_{T N, k}=\sum_{1 \leq n_{1}<\cdots<n_{k} \leq T N} \mathrm{E}\left[\prod_{\ell=1}^{k} \chi_{\beta_{N}, h_{N}}\left(\left|S_{n_{\ell}}\right|\right)\right], \quad \chi_{\beta_{N}, h_{N}}(x)=\mathrm{e}^{\psi_{\beta_{N}, h_{N}}(x)}-1 .
$$

In the sequel, $C, C^{\prime}<\infty$ denote absolute constants, possibly depending on $T, \hat{\beta}, \hat{h}$, that may change from line to line. Since $\beta_{N}, h_{N} \downarrow 0$, it follows from (1.21) that for $N \rightarrow \infty$ (recall that $\varphi$ is bounded):

$$
\text { uniformly in } x \in \mathbb{Z}: \quad \chi_{\beta_{N}, h_{N}}(x) \sim \psi_{\beta_{N}, h_{N}}(x) \sim \frac{1}{2} \beta_{N}^{2} \varphi(x)^{2}-h_{N} \varphi(x) \text {. }
$$

Since $\lim _{|x| \rightarrow \infty}|x|^{\vartheta} \varphi(x)=c \in(0, \infty)$ by $(1.44)$, we can bound

$$
\begin{aligned}
& \forall x \in \mathbb{Z}: \quad\left|\chi \beta_{N}, h_{N}(|x|)\right| \leq \bar{\chi}_{\beta_{N}, h_{N}}(|x|) \\
& \quad:=C\left(\frac{1}{2} \beta_{N}^{2}(1+|x|)^{-2 \vartheta}+h_{N}(1+|x|)^{-\vartheta}\right) .
\end{aligned}
$$

Next, recall the uniform upper bound in (1.42). For any $\gamma \neq 2(1-\alpha)$ we can bound

$$
\forall n \in \mathbb{N}: \quad \mathrm{E}\left[\left(1+\left|S_{n}\right|\right)^{-\gamma}\right] \leq \frac{C}{n^{1-\alpha}} \sum_{k \in \mathbb{Z}}(1+|k|)^{1-2 \alpha-\gamma} \mathrm{e}^{-\frac{|k|^{2}}{8 n}} \leq \frac{C^{\prime}}{n^{(1-\alpha) \wedge(\gamma / 2)}}
$$

(where $a \wedge b:=\min \{a, b\})$. Indeed,

- for $\gamma>2(1-\alpha)$ we drop the exponential and note that $\sum_{k \in \mathbb{Z}}(1+|k|)^{1-2 \alpha-\gamma}<\infty$;

- for $\gamma<2(1-\alpha)$ a Riemann sum approxmation shows that the sum can be bounded by a multiple of $n^{1-\alpha-\gamma / 2}$ (note that $\int_{0}^{\infty} x^{1-2 \alpha-\gamma} \mathrm{e}^{-x^{2} / 8} \mathrm{~d} x<\infty$ ).

It follows from (4.25)-(4.26) that

$$
\forall n, N \in \mathbb{N}: \quad \mathrm{E}\left[\bar{\chi}_{\beta_{N}, h_{N}}\left(\left|S_{n}\right|\right)\right] \leq C\left(\frac{\beta_{N}^{2}}{n^{(1-\alpha) \wedge \vartheta}}+\frac{h_{N}}{n^{(1-\alpha) \wedge(\vartheta / 2)}}\right) .
$$


Setting $n=N t$ with $t \in(0, T]$, and recalling that $\beta_{N}, h_{N}$ are given in (4.1)-(4.2), we obtain

$$
\begin{aligned}
& \forall N \in \mathbb{N}, \forall t \in(0, T] \cap N^{-1} \mathbb{N}: \\
& \mathrm{E}\left[\bar{\chi}_{\beta_{N}, h_{N}}\left(\left|S_{N t}\right|\right)\right] \leq \frac{C}{N}\left(\frac{1}{t^{(1-\alpha) \wedge \vartheta}}+\frac{1}{t^{(1-\alpha) \wedge(\vartheta / 2)}}\right) \leq \frac{C^{\prime}}{N} \frac{1}{t^{1-\alpha}} .
\end{aligned}
$$

Next, by (4.23) we can bound

$$
C_{T N, k} \leq \sum_{1 \leq n_{1}<\cdots<n_{k} \leq T N} \prod_{\ell=1}^{k} \mathrm{E}\left[\bar{\chi}_{\beta_{N}, h_{N}}\left(\left|S_{n_{\ell}-n_{\ell-1}}\right|\right)\right],
$$

because the function $\bar{\chi}_{\beta_{N}, h_{N}}(|x|)$ is decreasing in $|x|$ and the law $\mathrm{P}\left(\left|S_{n}\right| \in \cdot|| S_{n^{\prime}} \mid=m\right)$ stochastically dominates $\mathrm{P}\left(\left|S_{n}\right| \in \cdot \mid S_{n^{\prime}}=0\right)=\mathrm{P}\left(\left|S_{n-n^{\prime}}\right| \in \cdot\right)$ for all $m \geq 0$ (as can be seen via a coupling argument). Then, setting $n_{\ell}=N t_{\ell}$, via (4.28) we get

$$
\begin{aligned}
C_{T N, k} & \leq \frac{1}{N^{k}} \sum_{\substack{0<t_{1}<\cdots<t_{k} \leq T \\
\text { such that } \\
N t_{1}, \ldots, N t_{k} \in \mathbb{N}}} \prod_{\ell=1}^{k} \frac{C}{\left(t_{\ell}-t_{\ell-1}\right)^{1-\alpha}} \\
& \leq \int_{0<t_{1}<\cdots<t_{k}<T}\left\{\prod_{\ell=1}^{k} \frac{C}{\left(t_{\ell}-t_{\ell-1}\right)^{1-\alpha}}\right\} \mathrm{d} t_{1} \cdots \mathrm{d} t_{k} .
\end{aligned}
$$

This is the same bound as in (4.22), and hence the first inequaltiy in (4.13) is proved.

\subsection{Proof of (4.14)}

Intuitively, we can get (4.14) by substituting the asymptotic relation (4.24) into the definition (4.23) of $C_{T N, k}$ and performing a Riemann sum approximation, recalling (1.44). However, some care is required to properly implement this strategy.

1. Recall from (4.24) that $\chi_{\beta_{N}, h_{N}}(x) \sim \frac{\beta_{N}^{2}}{2} \varphi(x)^{2}-h_{N} \varphi(x)$ as $N \rightarrow \infty$, and by (1.44) the terms $\varphi(x)^{2}$ and $\varphi(x)$ decay polynomially as $|x|^{-\gamma}$ as $|x| \rightarrow \infty$, with $\gamma=2 \vartheta$ and $\gamma=\vartheta$, respectively. Fix $\varepsilon>0$ small and introduce an approximation $\chi_{\beta_{N}, h_{N}}^{\varepsilon}(x)$ of $\chi_{\beta_{N}, h_{N}}(x)$ in which each term is restricted to a relevant range: either $|x| \approx 1$ (more precisely, $|x| \leq \frac{1}{\varepsilon}$ ) or $|x| \approx \sqrt{N}$ (more precisely, $\varepsilon \sqrt{N} \leq|x| \leq \frac{1}{\varepsilon} \sqrt{N}$ ), depending on the decay exponent $\gamma$. Indeed, the bound in (4.26) on $\mathrm{E}\left[\left(1+\left|S_{N}\right|\right)^{-\gamma}\right] \leq$ tells us that

- for $\gamma>2(1-\alpha)$ the relevant contribution comes from $|x|=\left|S_{N}\right| \approx 1$;

- for $\gamma<2(1-\alpha)$ the relevant contribution comes from $|x|=\left|S_{N}\right| \approx \sqrt{N}$. 
This motivates the following definition:

$$
\begin{aligned}
& \chi_{\beta_{N}, h_{N}}^{\varepsilon}(x) \\
& \quad:= \begin{cases}\left\{\frac{1}{2} \beta_{N}^{2} \varphi(x)^{2}-h_{N} \varphi(x)\right\} 1_{\left\{\varepsilon \sqrt{N} \leq|x| \leq \frac{1}{\varepsilon} \sqrt{N}\right\}}, & \vartheta \in(0,1-\alpha), \\
\frac{1}{2} \beta_{N}^{2} \varphi(x)^{2} 1_{\left\{|x| \leq \frac{1}{\varepsilon}\right\}}-h_{N} \varphi(x) 1_{\left\{\varepsilon \sqrt{N} \leq|x| \leq \frac{1}{\varepsilon} \sqrt{N}\right\}}, & \vartheta \in(1-\alpha, 2(1-\alpha)), \\
\left\{\frac{1}{2} \beta_{N}^{2} \varphi(x)^{2}-h_{N} \varphi(x)\right\} 1_{\left\{|x| \leq \frac{1}{\varepsilon}\right\}}, & \vartheta \in(2(1-\alpha), \infty) .\end{cases}
\end{aligned}
$$

We correspondingly define an approximation $C_{T N, k}^{\varepsilon}$ of $C_{T N, k}$ (see (4.23)):

$$
C_{T N, k}^{\varepsilon}=\sum_{1 \leq n_{1}<\cdots<n_{k} \leq T N} \mathrm{E}\left[\prod_{\ell=1}^{k} \chi_{\beta_{N}, h_{N}}^{\varepsilon}\left(\left|S_{n_{\ell}}\right|\right)\right]
$$

This allows us to split the proof of (4.14) in two parts: For fixed $k \in \mathbb{N}$,

$$
\lim _{\varepsilon \downarrow 0} \limsup _{N \rightarrow \infty}\left|C_{T N, k}-C_{T N, k}^{\varepsilon}\right|=0, \quad \lim _{\varepsilon \downarrow 0} \lim _{N \rightarrow \infty} C_{T N, k}^{\varepsilon}=\hat{C}_{T, k}
$$

2. The first claim in (4.33) is a consequence of the bounds that we have derived in Sect. 4.3 for the proof of (4.13). Indeed, we can use a telescopic argument based on the bound

$$
\left|\prod_{\ell=1}^{k} a_{\ell}-\prod_{\ell=1}^{k} b_{\ell}\right| \leq \sum_{i=1}^{k}\left(\prod_{\ell=1}^{i-1}\left|a_{\ell}\right|\right)\left|a_{i}-b_{i}\right|\left(\prod_{\ell=i+1}^{k}\left|b_{\ell}\right|\right)
$$

to replace $a_{\ell}:=\chi_{\beta_{N}, h_{N}}\left(S_{n_{\ell}}\right)$ by $b_{\ell}:=\chi_{\beta_{N}, h_{N}}^{\varepsilon}\left(S_{n_{\ell}}\right)$ in the definition (4.23) of $C_{T N, k}$. Note that both $\left|a_{\ell}\right|$ and $\left|b_{\ell}\right|$ are bounded by $\bar{\chi}_{\beta_{N}, h_{N}}\left(\left|S_{n_{\ell}}\right|\right)$ defined in (4.25). Similarly, we can bound $\left|a_{i}-b_{i}\right|=\left|\left(\chi_{\beta_{N}, h_{N}}-\chi_{\beta_{N}, h_{N}}^{\varepsilon}\right)\left(S_{n_{i}}\right)\right| \leq \Delta \bar{\chi}_{\beta_{N}, h_{N}}^{\varepsilon}\left(\left|S_{n_{i}}\right|\right)$, where we define $\Delta \bar{\chi}_{\beta_{N}, h_{N}}^{\varepsilon}(|x|)$ to be $\bar{\chi}_{\beta_{N}, h_{N}}(|x|)$ in (4.25) with the terms $(1+|x|)^{-2 \vartheta}$ and $(1+|x|)^{-\vartheta}$ replaced as follows:

$$
(1+|x|)^{-\gamma} \rightsquigarrow \begin{cases}(1+|x|)^{-\gamma} 1_{\left\{|x|>\frac{1}{\varepsilon}\right\}}, & \gamma>2(1-\alpha) \\ (1+|x|)^{-\gamma} 1_{\{|x|<\varepsilon \sqrt{N}\} \cup\left\{|x|>\frac{1}{\varepsilon} \sqrt{N}\right\}}, & \gamma<2(1-\alpha) .\end{cases}
$$

This leads to

$$
\begin{aligned}
& \left|C_{T N, k}-C_{T N, k}^{\varepsilon}\right| \\
& \leq \sum_{i=1}^{k} \sum_{1 \leq n_{1}<\cdots<n_{k} \leq T N} \mathrm{E}\left[\Delta \bar{\chi}_{\beta_{N}, h_{N}}^{\varepsilon}\left(\left|S_{n_{i}}\right|\right) \times \prod_{\ell \in\{1, \ldots, k\} \backslash\{i\}} \bar{\chi}_{\beta_{N}, h_{N}}\left(\left|S_{n_{\ell}}\right|\right)\right] .
\end{aligned}
$$


3. Recall the bound (4.27) for $\mathrm{E}\left[\bar{\chi}_{\beta_{N}, h_{N}}\left(\left|S_{n}\right|\right)\right]$. A similar but improved bound holds for $\mathrm{E}\left[\Delta \bar{\chi}_{\beta_{N}, h_{N}}^{\varepsilon}(|x|)\right]$, namely,

$$
\begin{aligned}
\forall n, N \in \mathbb{N}: \quad & \mathrm{E}\left[\Delta \bar{\chi}_{\beta_{N}, h_{N}}^{\varepsilon}\left(\left|S_{n}\right|\right)\right] \leq C \eta_{\varepsilon}\left(\frac{\beta_{N}^{2}}{n^{(1-\alpha) \wedge \vartheta}}+\frac{h_{N}}{n^{(1-\alpha) \wedge(\vartheta / 2)}}\right) \\
& \text { with } \lim _{\varepsilon \downarrow 0} \eta_{\varepsilon}=0 .
\end{aligned}
$$

Indeed, by (4.26) and the lines following it, we can choose

$$
\eta_{\varepsilon}:= \begin{cases}\sum_{k>1 / \varepsilon}(1+|k|)^{1-2 \alpha-\gamma}, & \gamma>2(1-\alpha) \\ \int_{(0, \varepsilon) \cup(1 / \varepsilon, \infty)} x^{1-2 \alpha-\gamma} e^{-x^{2} / 8} \mathrm{~d} x, & \gamma<2(1-\alpha) .\end{cases}
$$

Consequently, arguing as in (4.28)-(4.29), we get the following improvement of (4.30):

$$
\left|C_{T N, k}-C_{T N, k}^{\varepsilon}\right| \leq k \eta_{\varepsilon} \int_{0<t_{1}<\cdots<t_{k}<T}\left\{\prod_{\ell=1}^{k} \frac{C}{\left(t_{\ell}-t_{\ell-1}\right)^{1-\alpha}}\right\} \mathrm{d} t_{1} \cdots \mathrm{d} t_{k}
$$

(set $n_{0}=0, x_{0}=0$ ), from which the first equation in (4.33) readily follows. With similar arguments we can show that in (4.32) we can further restrict the sum to $n_{\ell}-$ $n_{\ell-1} \geq \varepsilon N$ for all $\ell=1, \ldots, k$, with a negligible error as $N \rightarrow \infty$ followed by $\varepsilon \downarrow 0$ (we omit the details). We can thus update the definition (4.32) of $C_{T N, k}^{\varepsilon}$, where we also sum over space variables:

$$
C_{T N, k}^{\varepsilon}=\sum_{\substack{1 \leq n_{1}<\ldots<n_{k} \leq T N: \\ n_{\ell}-n_{\ell-1} \geq \varepsilon N \forall \ell=1, \ldots, k \\ x_{1}, \ldots, x_{k} \in \mathbb{N}_{0}}} \prod_{\ell=1}^{k} \mathrm{P}_{x_{\ell-1}}\left(\left|S_{n_{\ell}-n_{\ell-1}}\right|=x_{\ell}\right) \chi_{\beta_{N}, h_{N}}^{\varepsilon}\left(x_{\ell}\right)
$$

4. We are left with proving the second claim in (4.33). To do so, we distinguish between the three regimes in (4.31).

- First regime. In the regime $\vartheta \in(0,1-\alpha)$, the function $\chi_{\beta_{N}, h_{N}}^{\varepsilon}\left(x_{\ell}\right)$ restricts all variables $x_{\ell}$ in the diffusive range $\left\{\varepsilon \sqrt{N} \leq x \leq \frac{1}{\varepsilon} \sqrt{N}\right\}$ ("intermediate heights"). This allows us to apply the local limit theorem in (1.40), which we may rewrite as follows: Uniformly in $t \in[\varepsilon, T]$ and $\varepsilon \leq z^{\prime}, z \leq 1 / \varepsilon$,

$$
\mathrm{P}_{\sqrt{N} z^{\prime}}\left(\left|S_{N t}\right|=\sqrt{N} z\right) \sim \frac{2}{\sqrt{N}} g_{t}\left(z^{\prime}, z\right) 1_{\left\{\sqrt{N} z-\sqrt{N} z^{\prime}-N t \text { is even }\right\}}, \quad N \rightarrow \infty
$$

where we recall that $g_{t}\left(z^{\prime}, z\right)=g_{1}\left(z^{\prime} / \sqrt{t}, z / \sqrt{t}\right) / \sqrt{t}$. (For notational simplicity, we will ignore the parity constraint and remove the compensating factor 2.) Note that 
$\varphi(x) \sim c / x^{\vartheta}$ as $x \rightarrow \infty$ (see (1.44)). Therefore, by (4.1)-(4.4) and (4.31), uniformly in $\varepsilon \leq z \leq 1 / \varepsilon$,

$$
\chi_{\beta_{N}, h_{N}}^{\varepsilon}(\sqrt{N} z) \sim\left\{\frac{1}{2} \beta_{N}^{2} c^{2}(\sqrt{N} z)^{-2 \vartheta}-h_{N} c(\sqrt{N} z)^{-\vartheta}\right\} \sim \frac{\hat{\psi}_{\hat{\beta}, \hat{h}}(z)}{N} .
$$

Introducing macroscopic space-time variables $n_{\ell}=N t_{\ell}, x_{\ell}=\sqrt{N} z_{\ell}$, we get from (4.40) that

$$
\begin{aligned}
& C_{T N, k}^{\varepsilon} \sim \sum_{\substack{0<t_{1}<\cdots<t_{k} \leq T: \\
N t_{\ell} \in \mathbb{N} \text { and } t_{\ell}-t_{\ell-1} \geq \varepsilon \\
z_{1}, \ldots, z_{k} \in[0, \infty): \\
\sqrt{N} z_{\ell} \in \mathbb{Z} \text { and } \varepsilon \leq z_{\ell} \leq 1 / \varepsilon}} \prod_{\ell=1}^{k} \frac{g_{t_{\ell}-t_{\ell-1}}\left(z_{\ell-1}, z_{\ell}\right)}{\sqrt{N}} \frac{\hat{\psi}_{\hat{\beta}, \hat{h}}\left(z_{\ell}\right)}{N} \\
& \longrightarrow \int_{\substack{0<t_{1}<\cdots<t_{k} \leq T: \\
t_{\ell}-t_{\ell-1} \geq \varepsilon \forall \ell \\
z_{1}, \ldots, z_{k} \in[0, \infty): \\
\varepsilon \leq z_{\ell} \leq 1 / \varepsilon \forall \ell}} \prod_{\ell=1}^{k}\left\{g_{t_{\ell}-t_{\ell-1}}\left(z_{\ell-1}, z_{\ell}\right) \hat{\psi}_{\hat{\beta}, \hat{h}^{\prime}}\left(z_{\ell}\right)\right\} \mathrm{d} t_{1} \\
& \ldots \mathrm{d} t_{k} \mathrm{~d} z_{1} \ldots \mathrm{d} z_{k} \text {. }
\end{aligned}
$$

When we let $\varepsilon \downarrow 0$, the last integral converges to $\hat{C}_{T N, k}$ (see (4.10)). This proves the second claim in (4.33).

- Third regime. In the regime $\vartheta \in(2(1-\alpha), \infty)$, the function $\chi_{\beta_{N}, h_{N}}^{\varepsilon}\left(x_{\ell}\right)$ restricts all variables $x_{\ell}$ to the $O(1)$ range $\left\{x \leq \frac{1}{\varepsilon}\right\}$ ("low heights"). This allows us to apply the local limit theorem in (1.38), which we may rewrite as follows: Uniformly in $t \in[\varepsilon, T]$ and $0 \leq x^{\prime}, x \leq 1 / \varepsilon$,

$$
\mathrm{P}_{x^{\prime}}\left(\left|S_{N t}\right|=x\right) \sim \frac{2 c(x)}{N^{1-\alpha} t^{1-\alpha}} 1_{\left\{x-x^{\prime}-N t \text { is even }\right\}}, \quad N \rightarrow \infty,
$$

where we recall that $\hat{g}_{1}\left(x^{\prime} / \sqrt{n}, 0\right) \sim \hat{g}_{1}(0,0)=1$ (see (1.34)). (We again ignore the parity constraint and remove the compensating factor 2.) Moreover, it follows from (4.1)-(4.2) and (4.31) that uniformly for $x \leq 1 / \varepsilon$,

$$
\chi_{\beta_{N}, h_{N}}^{\varepsilon}(x)=\left\{\frac{1}{2} \beta_{N}^{2} \varphi(x)^{2}-h_{N} \varphi(x)\right\} \sim \frac{\frac{1}{2} \hat{\beta}^{2} \varphi(x)^{2}-\hat{h} \varphi(x)}{N^{\alpha}} .
$$


Introducing the macroscopic time variables $n_{\ell}=N t_{\ell}$, while keeping the microscopic space variables $x_{\ell}$, we get by (4.40) that

$$
\begin{aligned}
& C_{T N, k}^{\varepsilon} \sim \sum_{\substack{0<t_{1}<\ldots<t_{k} \leq T: \\
N t_{\ell} \in \mathbb{N} \text { and } t_{\ell}-t_{\ell-1} \geq \varepsilon \\
x_{1}, \ldots, x_{k} \in \mathbb{N}_{0}: \\
x_{\ell} \leq 1 / \varepsilon \forall \ell}} \prod_{\ell=1}^{k} \frac{c\left(x_{\ell}\right)}{N^{1-\alpha}\left(t_{\ell}-t_{\ell-1}\right)^{1-\alpha}} \frac{\frac{1}{2} \hat{\beta}^{2} \varphi\left(x_{\ell}\right)^{2}-\hat{h} \varphi\left(x_{\ell}\right)}{N^{\alpha}} \\
& \underset{N \rightarrow \infty}{\longrightarrow} \int_{\substack{0<t_{1}<\cdots<t_{k} \leq T: \\
t_{\ell}-t_{\ell-1} \geq \varepsilon \forall \ell}} \prod_{\ell=1}^{k} \frac{\left\{\frac{1}{2} \hat{\beta}^{2} \sum_{x \leq 1 / \varepsilon} c(x) \varphi(x)^{2}-\hat{h} \sum_{x \leq 1 / \varepsilon} c(x) \varphi(x)\right\}}{\left(t_{\ell}-t_{\ell-1}\right)^{1-\alpha}} \mathrm{d} t_{1} \\
& \ldots \mathrm{d} t_{k} \text {. }
\end{aligned}
$$

When we let $\varepsilon \downarrow 0$, the term in brackets converges to

$$
\frac{1}{2} \hat{\beta}^{2} \sum_{x \in \mathbb{N}_{0}} c(x) \varphi(x)^{2}-\hat{h} \sum_{x \in \mathbb{N}_{0}} c(x) \varphi(x)=: \frac{1}{2} \hat{\beta}^{2} c^{*}[\varphi]-\hat{h} c^{*}[\varphi] .
$$

Hence $C_{T N, k}^{\varepsilon}$ converges to $\hat{C}_{T N, k}$ (see (4.12)). This proves the second claim in (4.33).

- Second regime. In the regime $\vartheta \in(1-\alpha, 2(1-\alpha))$, the function $\chi_{\beta_{N}, h_{N}}^{\varepsilon}\left(x_{\ell}\right)$ is the sum of two terms with different restrictions, namely, $\left\{x \leq \frac{1}{\varepsilon}\right\}$ and $\{\varepsilon \sqrt{N} \leq x \leq$ $\left.\frac{1}{\varepsilon} \sqrt{N}\right\}$. Expanding the product over $\chi_{\beta_{N}, h_{N}}^{\varepsilon}\left(x_{\ell}\right)$ in (4.40), we obtain $2^{k}$ different terms, where every variable $x_{\ell}$ is subject to one of the two restrictions. Each of these terms can be analysed by arguing precisely as in the previous regimes:

- for $\left\{\varepsilon \sqrt{N} \leq x_{\ell} \leq \frac{1}{\varepsilon} \sqrt{N}\right\}$ we apply the local limit theorem in (4.41), introducing the macroscopic space variable $z_{\ell} \in[0, \infty)$ defined by $x_{\ell}=\sqrt{N} z_{\ell}$;

- for $\left\{x_{\ell} \leq \frac{1}{\varepsilon}\right\}$ we apply the local limit theorem in (4.44), keeping the microscopic variable $x_{\ell} \in \mathbb{N}_{0}$.

The choice of $\beta_{N}, h_{N}$ in (4.1)-(4.2) ensure that the appropriate Riemann factor appears, so that the sum defining $C_{N T, k}^{\varepsilon}$ converges to the integral in (4.10) as $N \rightarrow \infty$ followed by $\varepsilon \downarrow 0$. This integral defines $\hat{C}_{N T, k}$, with the recipe in (4.11). We omit the details, which are notationally tedious because of the need to specify the restriction for each variable $x_{\ell}$, but are ultimately straightforward.

Open Access This article is licensed under a Creative Commons Attribution 4.0 International License, which permits use, sharing, adaptation, distribution and reproduction in any medium or format, as long as you give appropriate credit to the original author(s) and the source, provide a link to the Creative Commons licence, and indicate if changes were made. The images or other third party material in this article are included in the article's Creative Commons licence, unless indicated otherwise in a credit line to the material. If material is not included in the article's Creative Commons licence and your intended use is not permitted by statutory regulation or exceeds the permitted use, you will need to obtain permission directly from the copyright holder. To view a copy of this licence, visit http://creativecommons.org/licenses/by/4.0/. 


\section{Appendix A: Equivalence}

In "Appendices A.1-A.3" we prove (1.16), (1.14) and (1.25), respectively. In "Appendix A.4" we show that the limits $N \rightarrow \infty$ and $T \rightarrow \infty$ in (4.5) may be interchanged.

\section{A.1. Proof of (1.16)}

For $N \in \mathbb{N}_{0}$ and $x \in \mathbb{N}_{0}$, define

$$
f_{x}(N):=\mathrm{P}_{x}\left(S_{n} \geq x \forall 0<n<N, S_{N}=x\right) .
$$

(If $S$ has period 2, then replace $N$ by $2 N$.) By superadditivity,

$$
\lim _{N \rightarrow \infty} \frac{1}{N} \log f_{x}(N)=: C_{x} \in(-\infty, 0] \text { exists. }
$$

For $z \in[0, \infty)$ and $x \in \mathbb{N}_{0}$, define

$$
F_{x}(z):=\sum_{N \in \mathbb{N}_{0}} z^{N} f_{x}(N)
$$

Then, clearly,

$$
C_{x}=0 \Longleftrightarrow F_{x}(1+\varepsilon)=\infty \forall \varepsilon>0 .
$$

By (1.6), we have $C_{0}=0$. Below we show that this implies $C_{x}=0 \forall x \in \mathbb{N}_{0}$. The proof is by induction on $x$.

Any path that starts at $x$, ends at $x$ and does not go below $x$ can be cut into pieces that zig-zag between $x$ and $x+1$ and pieces that start at $x+1$, end at $x+1$ and do not go below $x+1$. Hence we have

$$
F_{x}(z)=\frac{r_{x} z Q_{x}(z)}{1-F_{x+1}(z) Q_{x}(z)}, \quad Q_{x}(z):=\frac{1}{1-z r_{x}}, \quad r_{x}:=p_{x, x+1} p_{x+1, x}>0
$$

where $p_{x, x+1}:=\mathrm{P}\left(S_{1}=x+1 \mid S_{0}=x\right)$. We know that $F_{0}(1+\varepsilon)=\infty \forall \varepsilon>0$. We show that this implies $F_{x}(1+\varepsilon)=\infty \forall \varepsilon>0$ for every $x \in \mathbb{N}$. The proof is by induction on $x$.

Fix $x \in \mathbb{N}_{0}$ and suppose that $F_{x}(1+\varepsilon)=\infty \forall \varepsilon>0$. We argue by contradiction. Suppose that $F_{x+1}(1+\varepsilon)<\infty$ for $\varepsilon$ small enough. Because $r_{x}>0$ and $Q_{x}(1+\varepsilon)<$ $\infty$ for $\varepsilon$ small enough, it follows that $F_{x+1}(1+\varepsilon) Q_{x}(1+\varepsilon) \geq 1 \forall \varepsilon>0$, and by continuity that $F_{x+1}(1) Q_{x}(1) \geq 1$. To get the contradiction it therefore suffices to show that $F_{x+1}(1) Q_{x}(1)<1$. Now, because $\left(S_{n}\right)_{n \in \mathbb{N}_{0}}$ is recurrent, we have $F_{x+1}(1)=$ 
$p_{x+1, x+2}$. Because $Q_{x}(1)=1 /\left(1-r_{x}\right)$, it follows that

$$
F_{x+1}(1) Q_{x}(1)=\frac{p_{x+1, x+2}}{1-r_{x}}=\frac{p_{x+1, x+2}}{1-p_{x+1, x} p_{x, x+1}}=\mathrm{P}_{x+1}\left(S_{n} \geq x \forall n \in \mathbb{N}_{0}\right)<1,
$$

where for both the last equality and the inequality we again use recurrence.

To prove (1.16), note that for any $0 \leq M \leq N$,

$$
\begin{aligned}
& \mathrm{P}_{0}\left(S_{n} \geq M \forall M<n \leq N\right) \\
& \quad=\mathrm{P}_{0}\left(S_{n}=n \forall 0<n \leq M\right) \mathrm{P}_{M}\left(S_{n} \geq M \forall M<n \leq N\right) \\
& \geq\left[\prod_{x=0}^{M-1} p_{x, x+1}\right] f_{M}(N-M) .
\end{aligned}
$$

Hence

$$
\liminf _{N \rightarrow \infty} \frac{1}{N} \log \mathrm{P}_{0}\left(S_{n} \geq M \forall M<n \leq N\right) \geq C_{M} \quad \forall M \in \mathbb{N}_{0} .
$$

Since $C_{M}=0$ for all $M \in \mathbb{N}_{0}$, this implies (1.16).

\section{A.2. Proof of (1.14)}

Note that $Z_{N, \beta, h}^{\omega, \mathrm{c}} \leq Z_{N, \beta, h}^{\omega}$ for all $N \in \mathbb{N}_{0}$. Hence we only need to show that $Z_{N, \beta, h}^{\omega} \leq$ $\mathrm{e}^{o(N)} Z_{N, \beta, h}^{\omega, \mathrm{c}} \mathbb{P}$-a.s. Define the constrained partition function

$$
Z_{N, \beta, h}^{\omega, x, y}:=\mathrm{E}_{x}\left[\mathrm{e}^{\sum_{n=1}^{N}\left(\beta \omega_{n}-h\right) \varphi\left(S_{n}\right)} 1_{\left\{S_{n}=y\right\}}\right], \quad N \in \mathbb{N}_{0}, x, y \in \mathbb{Z}
$$

Then

$$
Z_{N, \beta, h}^{\omega}=\sum_{y \in \mathbb{Z}} Z_{N, \beta, h}^{\omega, 0, y}, \quad Z_{N, \beta, h}^{\omega, \mathrm{c}}=Z_{N, \beta, h}^{\omega, 0,0} .
$$

By (1.1), for every $\varepsilon>0$ there is an $M \in \mathbb{N}_{0}$ such that

$$
\left|\varphi(x)-\gamma^{+}\right| \leq \varepsilon, \quad x \geq M, \quad\left|\varphi(x)-\gamma^{-}\right| \leq \varepsilon, \quad x \leq-M .
$$

where we abbreviate $\gamma^{ \pm}=\lim _{x \rightarrow \pm \infty} \varphi(x)$ and recall that $\gamma^{+}=0, \gamma^{-} \in[0, \infty)$ according to (1.1). For $N \geq M$, split

$$
Z_{N, \beta, h}^{\omega}=\mathrm{I}+\mathrm{II}+\mathrm{III}
$$


with

$$
\mathrm{I}=\sum_{y \geq 2 M} Z_{N, \beta, h}^{\omega, 0, y}, \quad \mathrm{II}=\sum_{y \leq-2 M} Z_{N, \beta, h}^{\omega, 0, y}, \quad \mathrm{III}=\sum_{-2 M<y<2 M} Z_{N, \beta, h}^{\omega, 0, y} .
$$

We will show that all three terms are bounded by $\mathrm{e}^{o(N)} Z_{N, \beta, h}^{\omega, 0,0}$. For ease of notation we will pretend that the Markov chain has period 1. This is easily fixed when the period is 2 .

Consider I. Let $\sigma_{N}=\max \left\{0 \leq n \leq N: S_{n}=M\right\}$, and split

$$
\mathrm{I}=\sum_{M \leq m \leq N-M} \mathrm{E}_{0}\left[\mathrm{e}^{\sum_{n=1}^{N}\left(\beta \omega_{n}-h\right) \varphi\left(S_{n}\right)} 1_{\left\{S_{N} \geq 2 M\right\}} 1_{\left\{\sigma_{N}=m\right\}}\right] .
$$

Replace $\left(S_{m}, \ldots, S_{N}\right)$ by an $(N-M-m)$-step path from $M$ to $M$ that is everywhere $\geq M$, followed by an $M$-step downward path $(M, \ldots, 0)$. The cost of this replacement is at most

$$
\exp \left(\left[2 \varepsilon(N-M-m)+2 M\|\varphi\|_{\infty}\right]\left(\beta \Omega_{N}(\omega)+|h|\right)\right)
$$

for the weight factor, with $\Omega_{N}(\omega)=\max _{1 \leq n \leq N} \omega_{n}$, and at most

$$
\frac{\mathrm{P}_{M}\left(S_{n} \geq M \forall 0<n<N-M, S_{N} \geq 2 M\right)}{\mathrm{P}_{M}\left(S_{n} \geq M \forall 0<n<N-M-m, S_{N-M-m}=M\right) \Pi_{M}}
$$

for the path probability, with $\Pi_{M}:=\prod_{x=M}^{1} p_{x, x-1}$. The probability in the denominator equals $f_{M}(N-M-m)$, and so we get

$$
\mathrm{I} \leq \mathrm{e}^{\left[2 \varepsilon N+2 M\|\varphi\|_{\infty}\right]\left(\beta \Omega_{N}(\omega)+|h|\right)} \frac{\Pi_{M}^{-1}}{f_{M}(N-M-m)} Z_{N, \beta, h}^{\omega, 0,0} .
$$

Since $M$ is fixed, $\Pi_{M}>0,\|\varphi\|_{\infty}<\infty, \Omega_{N}=O(\log N) \mathbb{P}$-a.s. and $\max _{1 \leq n \leq N} 1 / f_{M}(n)=\mathrm{e}^{o(N)}$, we get $\mathrm{I}=\mathrm{e}^{o(N)} Z_{N, \beta, h}^{\omega, 0,0} \mathbb{P}$-a.s.

The argument for II is similar. For III we replace $\left(S_{N-M}, \ldots, S_{N}\right)$ by an $M$-step path from $S_{N-M}$ to 0 at a finite cost (depending on $M$ and $\Omega_{N}$ ).

\section{A.3. Proof of (1.25)}

Copy the argument in "Appendix A.2" starting from the analogue of (A.9):

$$
Z_{N, \beta, h}^{\text {ann, } x, y}:=\mathrm{E}_{x}\left[\mathrm{e}^{\sum_{n=1}^{N} \psi_{\beta, h}\left(S_{n}\right)} 1_{\left\{S_{n}=y\right\}}\right], \quad N \in \mathbb{N}_{0}, x, y \in \mathbb{Z} .
$$

Use that $\left\|\psi_{\beta, h}\right\|_{\infty}<\infty$, and that for every $\varepsilon>0$ there is an $M \in \mathbb{N}_{0}$ such that

$$
\left|\psi_{\beta, h}(x)-\eta^{+}\right| \leq \varepsilon, \quad x \geq M, \quad\left|\psi_{\beta, h}(x)-\eta^{-}\right| \leq \varepsilon, \quad x \leq-M,
$$

with $\eta^{ \pm}=\psi_{\beta, h}\left(\gamma^{ \pm}\right)=\log M\left(\beta \gamma^{ \pm}\right)-h \gamma^{ \pm}($recall $(1.21))$. 


\section{A.4. Interchange of limits}

The following two lemmas, which give us a sandwich for the annealed free energies in the discrete and in the continuous model, are the key to showing that the limits in (4.5) may be interchanged.

Lemma 4.2 For $M, N \in \mathbb{N}_{0}, \beta \in(0, \infty)$ and $h \in \mathbb{R}$, define

$$
\begin{aligned}
Z_{M, N, \beta, h}^{\mathrm{ann},-} & :=\min _{|x| \leq M} \sum_{|y| \leq M} Z_{N, \beta, h}^{\mathrm{ann}, x, y}, \\
Z_{N, \beta, h}^{\mathrm{ann},+} & :=\sup _{x \in \mathbb{Z}} \sum_{y \in \mathbb{Z}} \max _{0 \leq N^{\prime} \leq N} Z_{N^{\prime}, \beta, h}^{\mathrm{ann}, x, y} .
\end{aligned}
$$

Assume that $\lim _{|x| \rightarrow \infty} \varphi(x)=0$ (recall $\left.(1.44)\right)$. Then, for all $\beta \in(0, \infty)$ and $h \in \mathbb{R}$,

$$
\lim _{N \rightarrow \infty} \frac{1}{N} \log Z_{M, N, \beta, h}^{\mathrm{ann},-}=F^{\mathrm{ann}}(\beta, h)=\lim _{N \rightarrow \infty} \frac{1}{N} \log Z_{N, \beta, h}^{\mathrm{ann},+} \forall M \in \mathbb{N}_{0} .
$$

Proof Note that $Z_{M, N, \beta, h}^{\mathrm{ann},-} \leq Z_{N, \beta, h}^{\mathrm{ann},+}$ for all $M, N \in \mathbb{N}_{0}$. Hence we only need to show that $Z_{M, N, \beta, h}^{\text {ann, }} \leq \mathrm{e}^{o(N)} Z_{N, \beta, h}^{\text {ann,- }}$ as $N \rightarrow \infty$ for all $M \in \mathbb{N}_{0}$. Indeed, this will imply (A.21) because $F^{\mathrm{ann}}(\beta, h)=\lim _{N \rightarrow \infty} \frac{1}{N} \log Z_{N, \beta, h}^{\mathrm{ann}, 0,0}$, as proved in Appendix A.3. In what follows we fix $\beta, h$ and abbreviate $\mathcal{H}_{N}(S):=\sum_{n=1}^{N} \psi_{\beta, h}\left(S_{n}\right)$.

Recall (A.19), where $\eta^{ \pm}=0$ because $\gamma^{ \pm}=\lim _{x \rightarrow \pm \infty} \varphi(x)=0$ by assumption. Define $\tau_{M}:=\min \left\{n \in \mathbb{N}_{0}:\left|S_{n}\right| \leq M\right\}$ and split

$$
\mathrm{E}_{x}\left[\mathrm{e}^{\mathcal{H}_{N}(S)}\right]=\mathrm{I}_{x}+\mathrm{II}_{x}
$$

with

$$
\mathrm{I}_{x}=\mathrm{E}_{x}\left[\mathrm{e}^{\mathcal{H}_{N}(S)} 1_{\left\{\tau_{M}>N\right\}}\right], \quad \mathrm{II}_{x}=\mathrm{E}_{x}\left[\mathrm{e}^{\mathcal{H}_{N}(S)} 1_{\left\{\tau_{M} \leq N\right\}}\right] .
$$

For $x \geq M$ and $x \leq-M$,

$$
\begin{aligned}
\mathrm{I}_{x} & \leq \mathrm{e}^{\varepsilon N} \mathrm{P}_{x}\left(\tau_{M}>N\right) \leq \mathrm{e}^{\varepsilon N}, \\
\mathrm{II}_{x} & \leq \mathrm{E}_{x}\left[\mathrm{e}^{\varepsilon \tau_{M}} 1_{\left\{\tau_{M} \leq N\right\}} \max _{z= \pm M} \mathrm{E}_{z}\left[\mathrm{e}^{\mathcal{H}_{N-\tau_{M}}(S)}\right]\right] \\
& \leq \mathrm{e}^{\varepsilon N} \max _{|z| \leq M} \max _{0 \leq N^{\prime} \leq N} \mathrm{E}_{z}\left[\mathrm{e}^{\mathcal{H}_{N^{\prime}}(S)}\right] .
\end{aligned}
$$

Combining (A.22) and (A.24), we obtain

$$
Z_{N, \beta, h}^{\mathrm{ann},+} \leq\left(1+\mathrm{e}^{\varepsilon N}\right) \max _{|z| \leq M} \max _{0 \leq N^{\prime} \leq N} \mathrm{E}_{z}\left[\mathrm{e}^{\mathcal{H}_{N^{\prime}}(S)}\right] .
$$

For every $|x|,|y| \leq M$ we have

$$
\mathrm{E}_{x}\left[\mathrm{e}^{\mathcal{H}_{N^{\prime}}(S)}\right] \geq \Pi_{M} \mathrm{e}^{-M\left\|\psi_{\beta, h}\right\|_{\infty}} \mathrm{E}_{y}\left[\mathrm{e}^{\mathcal{H}_{N^{\prime}}(S)}\right]
$$


with $\Pi_{M}=\prod_{x=-M}^{M-1} p_{x, x+1} \wedge \prod_{x=M}^{-M+1} p_{x, x-1}$. Since $\Pi_{M}>0$, it follows that

$$
Z_{N, \beta, h}^{\mathrm{ann},+} \leq \mathrm{e}^{\varepsilon N+o(N)} \max _{0 \leq N^{\prime} \leq N} \mathrm{E}_{0}\left[\mathrm{e}^{\mathcal{H}_{N^{\prime}}(S)}\right], \quad N \rightarrow \infty .
$$

As shown in Appendix A.3,

$$
\mathrm{E}_{0}\left[\mathrm{e}^{\mathcal{H}_{N^{\prime}}(S)}\right]=Z_{N^{\prime}, \beta, h}^{\mathrm{ann}}=\mathrm{e}^{o\left(N^{\prime}\right)} Z_{N^{\prime}, \beta, h}^{\mathrm{ann}, 0,0}, \quad N^{\prime} \rightarrow \infty
$$

Since $N \mapsto \log Z_{N, \beta, h}^{\mathrm{ann}, 0,0}$ is super-additive, it follows that

$$
\max _{0 \leq N^{\prime} \leq N} \mathrm{E}_{0}\left[\mathrm{e}^{\mathcal{H}_{N^{\prime}}(S)}\right]=\mathrm{e}^{o(N)} \mathrm{E}_{0}\left[\mathrm{e}^{\mathcal{H}_{N}(S)}\right], \quad N \rightarrow \infty .
$$

Combining (A.27) and (A.29), we obtain

$$
Z_{N, \beta, h}^{\mathrm{ann},+} \leq \mathrm{e}^{\varepsilon N+o(N)} Z_{N, \beta, h}^{\mathrm{ann}, 0,0}, \quad N \rightarrow \infty .
$$

But

$$
Z_{N, \beta, h}^{\mathrm{ann}, 0,0} \leq \mathrm{E}_{0}\left[\mathrm{e}^{\mathcal{H}_{N}(S)} 1_{\left\{\left|S_{N}\right| \leq M\right\}}\right]
$$

Appealing once more to (A.26), we arrive at

$$
Z_{M, N, \beta, h}^{\mathrm{ann},+} \leq \mathrm{e}^{\varepsilon N+o(N)} Z_{M, N, \beta, h}^{\mathrm{ann},-}, \quad N \rightarrow \infty,
$$

which proves the claim because $\varepsilon>0$ is arbitrary.

Lemma 4.3 For $S, T \geq 0, \hat{\beta} \in(0, \infty)$ and $\hat{h} \in \mathbb{R}$, define

$$
\begin{aligned}
\hat{Z}_{S, T, \hat{\beta}, \hat{h}}^{\mathrm{ann},-} & :=\min _{|x| \leq S} \mathrm{E}_{x}\left[\mathrm{e}^{\int_{0}^{T} d t \hat{\psi}_{\hat{\beta}, \hat{h}}\left(X_{t}\right)} 1_{\left\{\left|X_{T}\right| \leq S\right\}}\right], \\
\hat{Z}_{T, \hat{\beta}, \hat{h}}^{\mathrm{ann},+} & :=\sup _{x \in \mathbb{R}} \max _{0 \leq T^{\prime} \leq T} \mathrm{E}_{x}\left[\mathrm{e}^{\int_{0}^{T^{\prime}} d t \hat{\psi}_{\hat{\beta}, \hat{h}}\left(X_{t}\right)}\right],
\end{aligned}
$$

with $\hat{\psi}_{\hat{\beta}, \hat{h}}$ defined in (4.4). Then, for all $\hat{\beta} \in(0, \infty)$ and $\hat{h} \in \mathbb{R}$,

$$
\lim _{T \rightarrow \infty} \frac{1}{T} \log \hat{Z}_{S, T, \hat{\beta}, \hat{h}}^{\mathrm{ann},-}=\hat{F}^{\mathrm{ann}}(\hat{\beta}, \hat{h})=\lim _{T \rightarrow \infty} \frac{1}{T} \log \hat{Z}_{T, \hat{\beta}, \hat{h}}^{\mathrm{ann},+} \forall S \in(0, \infty) .(\mathrm{A} .34)
$$

Proof The proof is similar to that of Lemma 4.2.

What makes Lemma 4.2 useful is that $N \mapsto \log Z_{M, N, \beta, h}^{\text {ann,- }}$ is superadditive for every $M \in \mathbb{N}_{0}$, while $N \mapsto \log Z_{N, \beta, h}^{\mathrm{ann},+}$ is subadditive. Consequently,

$$
\frac{1}{N} \log Z_{M, N, \beta, h}^{\mathrm{ann},-} \leq F^{\mathrm{ann}}(\beta, h) \leq \frac{1}{N} \log Z_{N, \beta, h}^{\mathrm{ann},+} \quad \forall M, N \in \mathbb{N} .
$$


Let $A, B$ be the pair of exponents appearing in part (a) of Theorems 1.6-1.8, i.e.,

$$
(A, B)= \begin{cases}((1-\vartheta) / 2,(2-\vartheta) / 2), & \vartheta \in(0,1-\alpha), \\ (\alpha / 2,(2-\vartheta) / 2), & \vartheta \in(1-\alpha, 2(1-\alpha)), \\ (\alpha / 2, \alpha), & \vartheta \in(2(1-\alpha), \infty) .\end{cases}
$$

Fix $T, S \in(0, \infty)$, in (A.35) replace $N$ by $T N$, and pick $M=S \sqrt{T N}, \beta=\hat{\beta} N^{-A}$, $h=\hat{h} N^{-B}$, to obtain

$$
\begin{aligned}
& \frac{1}{T} \log Z_{S \sqrt{T N}, T N, \hat{\beta} N^{-A}, \hat{h} N^{-B}}^{\mathrm{ann},-} \leq N F^{\mathrm{ann}}\left(\hat{\beta} N^{-A}, \hat{h} N^{-B}\right) \leq \frac{1}{T} \log Z_{T N, \hat{\beta} N^{-A}, \hat{h} N^{-B}}^{\mathrm{ann},+} \\
& \forall N \in \mathbb{N}, T, S \in(0, \infty) .
\end{aligned}
$$

Let $N \rightarrow \infty$ to obtain

$$
\begin{aligned}
& \frac{1}{T} \log \hat{Z}_{S, T, \hat{\beta}, \hat{h}}^{\mathrm{ann},-} \leq \liminf _{N \rightarrow \infty} N F^{\mathrm{ann}}\left(\hat{\beta} N^{-A}, \hat{h} N^{-B}\right) \\
& \quad \leq \limsup _{N \rightarrow \infty} N F^{\mathrm{ann}}\left(\hat{\beta} N^{-A}, \hat{h} N^{-B}\right) \leq \frac{1}{T} \log \hat{Z}_{T, \hat{\beta}, \hat{h}}^{\mathrm{ann},+} \forall T, S \in(0, \infty) .
\end{aligned}
$$

Here, the fact that

$$
\begin{aligned}
\hat{Z}_{S, T, \hat{\beta}, \hat{h}}^{\mathrm{ann},-} & =\lim _{N \rightarrow \infty} Z_{S \sqrt{T N}, T N, \hat{\beta} N^{-A}, \hat{h} N^{-B}}^{\mathrm{ann},-} \\
\hat{Z}_{T, \hat{\beta}, \hat{h}}^{\mathrm{ann},+} & =\lim _{N \rightarrow \infty} Z_{T N, \hat{\beta} N^{-A}, \hat{h} N^{-B}}^{\mathrm{ann},+}
\end{aligned}
$$

follows from the same argument as used in Section 4 to prove that

$$
\lim _{N \rightarrow \infty} Z_{T N, \hat{\beta} N^{-A}, \hat{h} N^{-B}}^{\mathrm{ann}}=\hat{Z}_{T, \hat{\beta}, \hat{h}}^{\mathrm{ann}}
$$

In particular, the scaling $M=S \sqrt{T N}$ fits well with the invariance principle in (1.37). Finally, let $T \rightarrow \infty$ in (A.38) and use Lemma 4.3, to obtain

$$
\lim _{N \rightarrow \infty} N F^{\text {ann }}\left(\hat{\beta} N^{-A}, \hat{h} N^{-B}\right)=\hat{F}^{\text {ann }}(\hat{\beta}, \hat{h})
$$

\section{Appendix B: Properties of the annealed scaling limit}

In this appendix we show that the annealed partition functions and the corresponding annealed free energies encountered in Theorems 1.6-1.8 are finite in each of the three regimes. We also give an explicit characterization of the annealed free energy and the annealed critical curve in the regime where $\vartheta \in(2(1-\alpha), \infty)$. 


\section{B.1. Finite free energies for the Bessel process}

For $\mu, T, \gamma \in[0, \infty)$, define

$$
\hat{Z}_{\mu, T}:=\hat{\mathrm{E}}_{0}\left[\exp \left(\mu \int_{0}^{T} \mathrm{~d} t X_{t}^{-\gamma}\right)\right], \quad \tilde{Z}_{\mu, T}:=\hat{\mathrm{E}}_{0}\left[\exp \left(\mu \hat{L}_{T}(0)\right)\right]
$$

We show that, for $0<\gamma<2(1-\alpha)$, these quantities grow at most exponentially as $T \rightarrow \infty$ :

$$
\begin{aligned}
& \forall \mu \in[0, \infty), \forall 0<\gamma<2(1-\alpha): \limsup _{T \rightarrow \infty} \frac{1}{T} \log \hat{Z}_{\mu, T}<\infty, \\
& \underset{T \rightarrow \infty}{\limsup } \frac{1}{T} \log \tilde{Z}_{\mu, T}<\infty .
\end{aligned}
$$

By Cauchy-Schwarz, this implies that all the free energies in Theorems 1.6-1.8 are finite.

We first focus on $\hat{Z}_{\mu, T}$, which we rewrite as

$$
\hat{Z}_{\mu, T}=1+\sum_{k \in \mathbb{N}} \mu^{k} \hat{C}_{k, T}
$$

with

$$
\hat{C}_{k, T}:=\int_{0 \leq t_{1}<\cdots<t_{k} \leq T} \mathrm{~d} t_{1} \cdots \mathrm{d} t_{k} \hat{\mathrm{E}}_{0}\left[\prod_{\ell=1}^{k} X_{t_{\ell}}^{-\gamma}\right]
$$

We use the Markov property at times $t_{1}, \ldots, t_{k}$ to estimate

$$
\begin{aligned}
\hat{\mathrm{E}}_{0}\left[\prod_{\ell=1}^{k} X_{t_{\ell}}^{-\gamma}\right] & =\int_{0 \leq x_{1}, \ldots, x_{k}<\infty} \prod_{\ell=1}^{k} \hat{\mathrm{P}}_{x_{\ell-1}}\left(X_{t_{\ell}-t_{\ell-1}} \in \mathrm{d} x_{\ell}\right) x_{\ell}^{-\gamma} \\
& \leq \int_{0 \leq x_{1}, \ldots, x_{k}<\infty} \prod_{\ell=1}^{k} \hat{\mathrm{P}}_{0}\left(X_{t_{\ell}-t_{\ell-1}} \in \mathrm{d} x_{\ell}\right) x_{\ell}^{-\gamma}
\end{aligned}
$$

where the inequality holds because $\hat{\mathrm{P}}_{0}\left(X_{t} \in \cdot\right)$ stochastically dominates $\hat{\mathrm{P}}_{x}\left(X_{t} \in \cdot\right)$ for any $x \geq 0$ (by a standard coupling argument and the fact that $X$ is a Markov process with continuous paths) and because $x \mapsto x^{-\gamma}$ is non-increasing. We thus obtain

$$
\hat{C}_{k, T} \leq \int_{0 \leq t_{1}<\cdots<t_{k} \leq T} \mathrm{~d} t_{1} \cdots \mathrm{d} t_{k} \prod_{\ell=1}^{k} \hat{\mathrm{E}}_{0}\left[X_{t_{\ell}-t_{\ell-1}}^{-\gamma}\right]
$$


with $t_{0}=0$. By diffusive scaling we have $\hat{\mathrm{E}}_{0}\left[X_{t}^{-\gamma}\right]=t^{-\gamma / 2} C$, with $C=\hat{\mathrm{E}}_{0}\left[X_{1}^{-\gamma}\right]<$ $\infty$ for $0<\gamma<2(1-\alpha)$ (recall (1.31)). The change of variables $t_{k}=T s_{k}$ yields

$$
\hat{Z}_{\mu, T} \leq 1+\sum_{k \in \mathbb{N}}\left(\mu C T^{1-\gamma / 2}\right)^{k} I_{k}(\gamma / 2)
$$

where, for $\vartheta \in(0,1)$,

$$
I_{k}(\vartheta):=\int_{0 \leq s_{1}<\cdots<s_{k} \leq 1} \mathrm{~d} s_{1} \cdots \mathrm{d} s_{k} \prod_{\ell=1}^{k}\left(s_{\ell}-s_{\ell-1}\right)^{-\vartheta}
$$

with $s_{0}=0$. Since

$$
\int_{0 \leq u_{1}<\ldots<u_{k-1} \leq 1} \mathrm{~d} u_{1} \cdots \mathrm{d} u_{k} \prod_{\ell=1}^{k}\left(u_{\ell}-u_{\ell-1}\right)^{-\vartheta}=\frac{\Gamma(1-\vartheta)^{k}}{\Gamma(k(1-\vartheta))}
$$

with $u_{0}=1$ and $u_{k}=1$ is the normalization of the Dirichlet distribution, after setting $s_{i}=s_{k} u_{i}$ for $i=1, \ldots, k-1$ in (B.8) we obtain

$$
\begin{aligned}
I_{k}(\vartheta) & =\int_{0 \leq s_{k} \leq 1} \mathrm{~d} s_{k} s_{k}^{k(1-\vartheta)-1} \frac{\Gamma(1-\vartheta)^{k}}{\Gamma(k(1-\vartheta))}=\frac{\Gamma(1-\vartheta)^{k}}{\Gamma(k(1-\vartheta)+1)} \\
& \leq \Gamma(1-\vartheta)^{k} \exp (-(1-\vartheta) k\{\log [(1-\vartheta) k]-1\}),
\end{aligned}
$$

where we have used Stirling's bound $\Gamma(x+1) \geq \mathrm{e}^{x(\log x-1)}$. Substitute $\vartheta=\gamma / 2$ and set

$$
A:=(1-\gamma / 2), \quad B:=\log (\mu C \Gamma(1-\gamma / 2))
$$

to obtain

$$
\begin{aligned}
\hat{Z}_{\mu, T} & \leq 1+\sum_{k \in \mathbb{N}} \exp (-A k[\log (A k)-1]+A k \log T+B k) \\
& =1+\sum_{k \in \mathbb{N}} \exp \left(T \cdot \frac{k}{T}\left\{-A\left[\log \left(A \frac{k}{T}\right)-1\right]+B\right\}\right)
\end{aligned}
$$

We now set $x:=k / T$ and note by direct computation that, with $\bar{x}:=A^{-1} \mathrm{e}^{B / A}$,

$$
\sup _{x \in[0, \infty)} x\{-A[\log (A x)-1]+B\}=\bar{x}\{-A[\log (A \bar{x})-1]+B\}=\mathrm{e}^{B / A}
$$

Therefore the leading contribution to the sum in (B.12) is given by $k \approx \bar{x} T$ (more precisely, the sum restricted to $0 \leq k<2 \bar{x} T$ is at most $2 \bar{x} T \exp \left(T \mathrm{e}^{B / A}\right)$, while the 
contribution of the remaining terms with $k \geq 2 \bar{x} T$ is negligible). It follows that

$$
\limsup _{T \rightarrow \infty} \frac{1}{T} \log \hat{Z}_{\mu, T} \leq \mathrm{e}^{B / A}=[\mu C \Gamma(1-\gamma / 2)]^{\frac{1}{1-\gamma / 2}}<\infty .
$$

We next focus on $\tilde{Z}_{\mu, T}$, which equals

$$
\tilde{Z}_{\mu, T}=1+\sum_{k \in \mathbb{N}} \mu^{k} \bar{C}_{k, T}
$$

with

$$
\bar{C}_{k, T}:=\frac{1}{k !} \hat{\mathrm{E}}\left[\hat{L}_{T}(0)^{k}\right]
$$

We recall from $(1.35)$ that we can write $\hat{L}_{T}(0)=\lim _{\varepsilon \downarrow 0} \hat{L}_{T}^{\varepsilon}(0)$ in probability, where

$$
\hat{L}_{T}^{\varepsilon}(0):=\frac{c_{\alpha}}{\varepsilon^{2(1-\alpha)}} \int_{0}^{T} \mathrm{~d} s 1_{\left\{X_{s} \in(0, \varepsilon)\right\}}, \quad \text { with } \quad c_{\alpha}:=\frac{\Gamma(2-\alpha)}{2^{\alpha-1}}
$$

Let us focus on $\hat{L}_{T}^{\varepsilon}(0)$ for a moment. By explicit computation, for any $k \in \mathbb{N}$ (with $\left.t_{0}:=0\right)$

$$
\begin{aligned}
\frac{1}{k !} \hat{\mathrm{E}}\left[\hat{L}_{T}^{\varepsilon}(0)^{k}\right] & =\frac{c_{\alpha}^{k}}{\varepsilon^{2(1-\alpha) k}} \int_{0 \leq t_{1}<\cdots<t_{k} \leq T} \mathrm{~d} t_{1} \cdots \mathrm{d} t_{k} \hat{\mathrm{P}}_{0}\left(\bigcap_{\ell=1}^{k}\left\{X_{t_{\ell}} \in(0, \varepsilon)\right\}\right) \\
& \leq \frac{c_{\alpha}^{k}}{\varepsilon^{2(1-\alpha) k}} \int_{0 \leq t_{1}<\cdots<t_{k} \leq T} \mathrm{~d} t_{1} \cdots \mathrm{d} t_{k} \prod_{\ell=1}^{k} \hat{\mathrm{P}}_{0}\left(X_{t_{\ell}-t_{\ell-1}} \in(0, \varepsilon)\right) \\
& \leq \int_{0 \leq t_{1}<\cdots<t_{k} \leq T} \mathrm{~d} t_{1} \cdots \mathrm{d} t_{k} \prod_{\ell=1}^{k}\left(t_{\ell}-t_{\ell-1}\right)^{-(1-\alpha)}=T^{\alpha} I_{k}(1-\alpha),
\end{aligned}
$$

where the first inequality holds because $\hat{\mathrm{P}}_{0}\left(X_{t} \in \cdot\right)$ stochastically dominates $\hat{\mathrm{P}}_{x}$ $\left(X_{t} \in \cdot\right.$ ) for any $x \geq 0$, while the last inequality holds by (1.32) and the definition (B.8) of $I_{k}(\cdot)$. This shows that, as $\varepsilon \downarrow 0, \hat{L}_{T}^{\varepsilon}(0)$ is uniformly bounded in $L^{k}$, for any $k \in \mathbb{N}$. By uniform integrability, we can therefore exchange $\lim _{\varepsilon \downarrow}$ and $\hat{E}$ to get, recalling (B.16),

$$
\bar{C}_{k, T}=\lim _{\varepsilon \downarrow 0} \frac{1}{k !} \hat{\mathrm{E}}\left[\hat{L}_{T}^{\varepsilon}(0)^{k}\right]=T^{\alpha} I_{k}(1-\alpha),
$$


where the last equality holds because the inequality in (B.18) becomes sharp as $\varepsilon \downarrow 0$. Hence

$$
\begin{aligned}
\tilde{Z}_{\mu, T} & =1+\sum_{k \in \mathbb{N}} \mu^{k} \int_{0 \leq t_{1}<\cdots<t_{k} \leq T} \mathrm{~d} t_{1} \cdots \mathrm{d} t_{k} \prod_{\ell=1}^{k}\left(t_{\ell}-t_{\ell-1}\right)^{-(1-\alpha)} \\
& =1+\sum_{k \in \mathbb{N}}\left(\mu T^{\alpha}\right)^{k} I_{k}(1-\alpha)
\end{aligned}
$$

The steps in (B.10)-(B.14) (with $\vartheta=1-\alpha$ instead of $\vartheta=\gamma / 2$ ) show that not only $\tilde{Z}_{\mu, T}<\infty$ for all $\mu, T$, but also $\lim _{T \rightarrow \infty} \frac{1}{T} \log \tilde{Z}_{\mu, T}<\infty$ for all $\mu$.

\section{B.2. Formula for the annealed free energy}

To compute the annealed free energy $\hat{F}(\hat{\beta}, \hat{h})$ in the regime $\vartheta \in(2(1-\alpha), \infty)$, we use the first line in (B.20) to compute the Laplace transform of $\tilde{Z}_{\mu, T}$. Writing $\mathrm{e}^{-\lambda T}=\left(\prod_{\ell=1}^{k} \mathrm{e}^{-\lambda\left(t_{\ell}-t_{\ell-1}\right)}\right) \mathrm{e}^{-\lambda\left(T-t_{k}\right)}$ for $\lambda \geq 0$, we get

$$
\int_{0}^{\infty} \mathrm{d} T \lambda \mathrm{e}^{-\lambda T} \tilde{Z}_{\mu, T}=1+\sum_{k \in \mathbb{N}}\left(\mu \int_{0}^{\infty} t^{\alpha-1} e^{-\lambda t} \mathrm{~d} t\right)^{k}=\frac{1}{1-\mu \lambda^{-\alpha} \Gamma(\alpha)}
$$

Hence

$$
F(\mu)=\lim _{T \rightarrow \infty} \frac{1}{T} \log \tilde{Z}_{\mu, T}= \begin{cases}(\mu \Gamma(\alpha))^{1 / \alpha}, & \mu \geq 0 \\ 0, & \mu<0\end{cases}
$$

which proves (1.57).

\section{Appendix C: Localization criterion for the annealed model}

In this appendix we take a closer look at the criterion in (1.62) and show that it does not depend on the starting point of the walk. For the purpose of this appendix, let $S=\left(S_{n}\right)_{n \in \mathbb{N}_{0}}$ be any recurrent Markov chain on a countable space $E$, and let $\psi: E \rightarrow \mathbb{R}$ be an arbitrary function. Denote by $\tau^{x}:=\min \left\{n \in \mathbb{N}: S_{n}=x\right\}$ the first return time of $S$ to $x$. Define

$$
A_{x}:=\sum_{m \in \mathbb{N}} \mathrm{E}_{x}\left[\mathrm{e}^{\sum_{n=1}^{m} \psi\left(S_{n}\right)} 1_{\left\{\tau^{x}=m\right\}}\right] \in(0, \infty] .
$$

We will prove the following property:

$$
\forall x, y: \quad A_{x}>1 \Longleftrightarrow A_{y}>1 .
$$


It is convenient to introduce the following shorthand notation, for (possibly random) $\sigma \in \mathbb{N}$ :

$$
\mathcal{H}(\sigma):=\sum_{n=1}^{\sigma} \psi\left(S_{n}\right)
$$

so that we may simply write $A_{x}=\mathrm{E}_{x}\left[\mathrm{e}^{\mathcal{H}\left(\tau^{x}\right)}\right]$. Given an arbitrary $y$, we can split this expected value according to the two complementary events $\left\{\tau^{x}<\tau^{y}\right\}$ and $\left\{\tau^{y}<\tau^{x}\right\}$ :

$$
A_{x}=\mathrm{E}_{x}\left[\mathrm{e}^{\mathcal{H}\left(\tau^{x}\right)} 1_{\left\{\tau^{x}<\tau^{y}\right\}}\right]+\mathrm{E}_{x}\left[\mathrm{e}^{\mathcal{H}\left(\tau^{x}\right)} 1_{\left\{\tau^{y}<\tau^{x}\right\}}\right]
$$

The second term can be expanded by summing over all visits of $S$ to $y$ that precede the first return to $x$. If we define

$$
B_{x y}:=\mathrm{E}_{x}\left[\mathrm{e}^{\mathcal{H}\left(\tau^{x}\right)} 1_{\left\{\tau^{x}<\tau^{y}\right\}}\right], \quad C_{x y}:=\mathrm{E}_{x}\left[\mathrm{e}^{\mathcal{H}\left(\tau^{y}\right)} 1_{\left\{\tau^{y}<\tau^{x}\right\}}\right]
$$

then by the strong Markov property we get

$$
A_{x}=B_{x y}+\sum_{\ell=0}^{\infty} C_{x y}\left(B_{y x}\right)^{\ell} C_{y x}=B_{x y}+\frac{C_{x y} C_{y x}}{1-B_{y x}},
$$

with the convention that $A_{x}=\infty$ if $B_{y x} \geq 1$. Exchanging the roles of $x$ and $y$, we get

$$
A_{y}=B_{y x}+\frac{C_{x y} C_{y x}}{1-B_{x y}}
$$

We are now ready to prove (C.2). Fix $x, y$. We show that if $A_{x} \leq 1$, then also $A_{y} \leq 1$. To simplify the notation, we abbreviate $b:=B_{x y}, b^{\prime}:=B_{y x}$ and $c:=C_{x y} C_{y x}$, so that

$$
A_{x}=b+\frac{c}{1-b^{\prime}}, \quad A_{y}=b^{\prime}+\frac{c}{1-b},
$$

with the convention that the ratios equal $\infty$ if $b^{\prime} \geq 1$, respectively, $b \geq 1$. Assume that $A_{x} \leq 1$. Then we must have $b^{\prime}<1$ and the formula $A_{x}=b+\frac{c}{1-b^{\prime}}$ applies, which shows that also $b<1$ (because $c>0$ ). Hence we can write

$$
\begin{aligned}
A_{y} & =b^{\prime}+\frac{c}{1-b}=b^{\prime}+\frac{1-b^{\prime}}{1-b} \frac{c}{1-b^{\prime}} \\
& =b^{\prime}+\frac{1-b^{\prime}}{1-b}\left(A_{x}-b\right) \leq b^{\prime}+\frac{1-b^{\prime}}{1-b}(1-b)=1
\end{aligned}
$$

i.e., $A_{y} \leq 1$. 


\section{References}

1. Alexander, K.S.: The effect of disorder on polymer depinning transitions. Commun. Math. Phys. 279, 117-146 (2008)

2. Alexander, K.S.: Excursions and local limit theorems for Bessel-like random walks. Electron. J. Probab. 16, 1-44 (2011)

3. Alexander, K.S., Zygouras, N.: Quenched and annealed critical points in polymer pinning models. Commun. Math. Phys. 291, 659-689 (2009)

4. Berger, Q., Caravenna, F., Poisat, J., Sun, R., Zygouras, N.: The critical curve of the random pinning and copolymer models at weak coupling. Commun. Math. Phys. 326, 507-530 (2014)

5. Berger, Q., Giacomin, G., Lacoin, H.: Disorder and critical phenomena: the $\alpha=0$ copolymer model. To appear in Probab. Theory Relat. Fields, arXiv:1712.02261

6. Berger, Q., Lacoin, H.: Pinning on a defect line: characterization of marginal disorder relevance and sharp asymptotics for the critical point shift. J. Inst. Math. Jussieu 17, 305-346 (2018)

7. Birkner, M.: Conditional large deviations for a sequence of words. Stoch. Proc. Appl. 118, 703-729 (2008)

8. Birkner, M., Greven, A., den Hollander, F.: Quenched LDP for words in a letter sequence. Probab. Theory Relat. Fields 148, 403-456 (2010)

9. Bodineau, T., Giacomin, G.: On the localization transition of random copolymers near selective interfaces. J. Stat. Phys. 117, 17-34 (2004)

10. Bolthausen, E., den Hollander, F.: Localization transition for a polymer near an interface. Ann. Probab. 25, 1334-1366 (1997)

11. Bolthausen, E., den Hollander, F., Opoku, A.A.: A copolymer near a selective interface: variational characterization of the free energy. Ann. Probab. 43, 875-933 (2015)

12. Caravenna, F., Giacomin, G.: The weak coupling limit of disordered copolymer models. Ann. Probab. 38, 2322-2378 (2010)

13. Caravenna, F., Giacomin, G., Toninelli, F.L.: Copolymers at selective interfaces: settled issues and open problems. In: Probability in Complex Physical Systems, vol. 11, Springer Proceedings in Mathematics, pp. 289-311 (2012)

14. Caravenna, F., den Hollander, F.: A generalized smoothing inequality for disordered polymers. Electr. Commun. Probab. 18(76), 1-15 (2013)

15. Caravenna, F., Sun, R., Zygouras, N.: Polynomial chaos and scaling limits of disordered systems. J. Eur. Math. Soc. 19, 1-65 (2017)

16. Caravenna, F., Toninelli, F.L., Torri, N.: Universality for the pinning model in the weak coupling regime. Ann. Probab. 45, 2154-2209 (2017)

17. Cheliotis, D., den Hollander, F.: Variational characterization of the critical curve for pinning of random polymers. Ann. Probab. 41, 1767-1805 (2013)

18. Koralov, M., Cranston, L., Molchanov, S., Vainberg, B.: Continuous model for homopolymers. J. Funct. Anal. 256, 2656-2696 (2009)

19. Dembo, A., Zeitouni, O.: Large Deviations Techniques and Applications, 2nd edn. Springer, Berlin (1998)

20. Derrida, B., Giacomin, G., Lacoin, H., Toninelli, F.L.: Fractional moment bounds and disorder relevance for pinning models. Commun. Math. Phys. 287, 867-887 (2009)

21. Giacomin, G.: Random Polymer Models. Imperial College Press, London (2007)

22. Giacomin, G.: Disorder and critical phenomena through basic probability models. École d'Été de Probabilités de Saint-Flour XL-2010. Lecture Notes in Mathematics, vol. 2025. Springer (2011)

23. Giacomin, G., Lacoin, H., Toninelli, F.L.: Marginal relevance of disorder for pinning models. Commun. Pure Appl. Math. 63, 233-265 (2010)

24. den Hollander, F.: Random polymers. École d'Été de Probabilités de Saint-Flour XXXVII-2007. Lecture Notes in Mathematics, vol. 1974. Springer (2009)

25. Lacoin, H.: Influence of spatial correlation for directed polymers. Ann. Probab. 39, 139-175 (2011)

26. Lamperti, J.: A new class of probability limit theorems. J. Math. Mech. 11, 749-772 (1962)

27. Revuz, D., Yor, M.: Continuous Martingales and Brownian Motion, 3rd edn. Springer, Berlin (1999)

Publisher's Note Springer Nature remains neutral with regard to jurisdictional claims in published maps and institutional affiliations. 